M. Itô

Nagoya Math. J.

Vol. 55 (1974), 111-144

\title{
SUR LES CÔNES CONVEXES DE RIESZ ET LES NOYAUX DE CONVOLUTION COMPLÈTEMENT SOUS-HARMONIQUES
}

\author{
MASAYUKI ITÔ
}

\section{Introduction}

Soit $X$ un groupe abélien localement compact et dénombrable à l'infini; $\xi$ sera sa mesure de Haar. Dans les articles précédents [10] et [11], pour un noyau de convolution de Hunt $N$ sur $X$, nous avons défini la famille sous-ordonnée $H(N ; X)$ au noyau $N$, qui est une large classe de noyaux de convolution de Hunt sur $X$ définie par $N$ et la totalité des noyaux de convolution de Hunt bornés sur la droite réelle $\boldsymbol{R}$ portés par $\boldsymbol{R}^{+}=\{t \in \boldsymbol{R} ; t \geqq 0\}$.

Dans l'autre article [7], nous avons montré le théorème suivant:

Supposons que, pour un noyau de convolution $N$ sur $X$, il existe la résolvante $\left(N_{p}\right)_{p \geqq 0}$ associée au noyau $N$. Pour une mesure de Radon positive $\nu$ quelconque sur $\boldsymbol{R}^{+}$et pour une constante non-négative $c$, le noyau de convolution $c \varepsilon+\int N_{p} d \nu(p)$ sur $X$ satisfait au principe de domination dès que cela a un sens, où $\varepsilon$ est la mesure de Dirac à l'origine dans $X$.

Cette proposition entraîne que la somme de puissances fractionnaires de $N$ satisfait aussi au principe de domination. D'autre part, la formule de Riesz concernant les potentiels de Riesz-Frostman est bien connue et très utile. Ces deux résultats portera naturellement la définition du cône convexe de Riesz relatif à un noyau de convolution de Hunt sur $X$.

Notre premier but sera de montrer le théorème suivant:

Pour un noyau de convolution de Hunt $N$ sur $X$, il existe uniquement un cône convexe de Riesz $C_{R}(N)$ relatif au noyau $N$ contenu dans $\bar{H}(N ; X)=H(N ; X) \cup\{0\}$. Soit $\left(N_{p}\right)_{p \geqq 0}$ la résolvante associée au noyau $N$; alors $C_{R}(N)$ est la totalité des noyaux de convolution sur $X$ de la

Received March 16, 1973. 
forme $c \varepsilon+\int N_{p} d \nu(p)$, où $c$ et $\nu$ sont respectivement une constante nonnégative et une mesure de Radon positive sur $\boldsymbol{R}^{+}$. On obtiendra en même temps $C_{R}(N)=\cap C(N)$, où $C(N)$ est un cône convexe de Riesz relatif au noyau $N$.

La deuxième part de cet article sera consacrée à la détermination explicite d'un cône convexe de Riesz relatif au noyau newtonien. Soit $\boldsymbol{R}^{n}$ l'espace euclidien à $n(\geqq 1)$ dimensions; on notera $|x|$ la distance entre $x$ et l'origine. Un noyau de convolution $N$ sur $\boldsymbol{R}^{n}$ sera dit complètement sous-harmonique en dehors de l'origine si l'on a, quel que soit $k$ un entier $\geqq 1, \Delta^{k} N \geqq 0$ au sens des distributions en dehors de l'origine, où $\Delta$ est l'opérateur de Laplace sur $R^{n}$ et $\Delta^{k}=\Delta^{k-1} \Delta(k \geqq 2)$. Pour un nombre $p>0$, désignons par $G_{p}$ le noyau de convolution sur $\boldsymbol{R}^{n}$ dont la transformée de Fourier est égale à $\left(p+|x|^{2}\right)^{-1}$. Si un noyau de convolution $N$ sur $\boldsymbol{R}^{n}$ est complètemet sous-harmonique en dehors de l'origine et invariant par rotations, alors il satisfait au principe complet $\mathrm{du}$ maximum et il est de la forme $N=c_{1}+c_{2} \varepsilon+\int G_{p} d \nu(p)$, où $c_{i}(i=1,2)$ et $\nu$ sont respectivement une constante non-négative et une mesure de Radon positive sur $\boldsymbol{R}^{+}$. Soit $S_{r, 0}\left(\boldsymbol{R}^{n}\right)$ la totalité des noyaux de convolution sur $\boldsymbol{R}^{n}$ complètement sous-harmoniques en dehors de l'origine, invariants par rotations et s'annulant à l'infini ; alors si $n \geqq 3, S_{r, 0}\left(\boldsymbol{R}^{n}\right)$ sera un seul cône convexe de Riesz relatif au noyau newtonien sur $\boldsymbol{R}^{n}$ et constitué par de noyaux de convolution sur $\boldsymbol{R}^{n}$ invariants par rotations.

\section{Préliminaires}

Dans cette note, on notera:

$C_{K}(X)$ l'espace vectoriel topologique usuel des fonctions finies et continues dans $X$ à support compact,

$M_{K}(X)$ l'espace vectoriel topologique usuel des fonctions $\xi$-mesurables et bornées dans $X$ à valeurs réelles et à support compact,

$L_{10 \mathrm{c}}(X)$ l'espace vectoriel topologique usuel des fonctions localement $\xi$-sommables dans $X$ à valeurs réelles.

$C_{K}^{+}(X), M_{K}^{+}(X)$ et $L_{\text {loc }}^{+}(X)$ sont respectivement leur sous-ensembles des fonctions non-négatives.

Dans la théorie du potentiel, un noyau de convolution $N$ sur $X$ signifie une mesure de Radon positive dans $X$. On appelle le noyau 
adjoint $\check{N}$ de $N$ la mesure symétrisant avec $N$ par rapport à l'origine. Si, en particulier, $N=\check{N}$, il est dit symétrique. Si $N$ est de la forme $d N=K(x) d \xi(x)$, où $K \in L_{\text {loc }}^{+}(X)$, alors $N$ est un noyau-fonction de convolution sur $X$, et dans ce cas, on utilisera souvent $K$ au lieu de $N$. On dit qu'un noyau de convolution $N$ sur $X$ est borné (resp. s'annule à l'infini) si, quelle que soit $f$ de $C_{K}(X)$, la fonction $N * f$ est bornée sur $X$ (resp. $N * f(x)$ tend vers 0 avec $x \rightarrow \infty$ ).

Soit $N$ un noyau de convolution sur $X$. Pour une mesure de Radon réelle $\mu$ dans $X, N * \mu$ s'appelle le $N$-potentiel de $\mu$ dès que cette convolution a un sens. S'il est absolument continu par rapport à $\xi$, sa densité s'écrira $N \mu$. Pour une fonction $f$ de $L_{100}(X)$, on notera $N * f$ et $N f$ au lieu de $N *(f \xi)$ et $N(f \xi)$ dès que ceux sont définis. Pour une fonction borélienne $f$ dans $X$, on peut supposer $N f=N * f$ dès que, quel que soit $x$ de $X, \tau_{x} f$ est $\check{N}$-sommable, où $\tau_{x} f$ est la fonction obtenue de $f$ par la translation $x$.

On dit qu'un noyau de convolution $N$ sur $X$ satisfait au principe de domination (resp. au principe complet de maximum) si, quelles que soient $f$ et $g$ de $C_{K}^{+}(X)$, l'implication suivante a lieu :

$$
\begin{gathered}
N f \leqq N g(\text { resp. } N f \leqq N g+1) \operatorname{sur} \operatorname{supp}(f) \\
\Rightarrow N f \leqq N g(\text { resp. } N f \leqq N g+1) \operatorname{sur} X,
\end{gathered}
$$

où supp $(f)$ désigne le support de $f$. Si $N$ est borné, alors les deux principes pour $N$ sont équivalents (cf. [8]). On connaît bien que le principe de domination pour $N$ (resp. le principe complet du maximum pour $N$ ) est équivalent au principe de domination pour $\check{N}$ (resp. au principe complet du maximum pour $\check{N}$ ). D'autre part, il est aussi connu que le principe de domination pour $N$ (resp. le principe complet du maximum pour $N$ ) est équivalent au principe du balayage pour $N$ (resp. au principe du balayage avec diminution de masse pour $N$ ). On dit que $N$ satisfait au principe du balayage si, pour une mesure de Radon positive $\mu$ dans $X$ et pour un ouvert relativement compact $\omega$ de $X$, il existe une mesure de Radon positive $\mu_{\omega}^{\prime}$ dans $X$ portée par $\bar{\omega}$ telle que $N * \mu$ $\geqq N * \mu_{\omega}^{\prime}$ dans $X, N * \mu=N * \mu_{\omega}^{\prime}$ dans $\omega$ et, quelle que soit $\mu^{\prime \prime}$ une mesure de Radon positive dans $X$ portée par $\bar{\omega}, N * \mu_{\omega}^{\prime} \leqq N * \mu^{\prime \prime}$ dans $X$ dès que $N * \mu^{\prime \prime} \geqq N * \mu$ dans $\omega$. On dit que $\mu_{\omega}^{\prime}$ est une mesure balayée de $\mu$ sur $\omega$ relativement au noyau $N$. Si $N$ satisfait au principe du balayage et, quels que soient $\mu$ une mesure de Radon positive dans $X$ à support com- 
pact et $\omega$ un ouvert relativement compact de $X, \int d \mu_{\omega}^{\prime} \leqq \int d \mu$, alors on dit que $N$ satisfait au principe du balayage avec diminution de masse.

Un noyau de convolution $N$ sur $X$ satisfait au principe de domination et vérifie la condition supplémentaire "régularité" si et seulement s'il existe la résolvante associée au noyau $N$ (cf. [9]). Une famille $\left(N_{p}\right)_{p>0}$ de noyaux de convolution sur $X$ s'appelle une résolvante si, quels que soient $p>0$ et $q>0$,

$$
N_{p}-N_{q}=(q-p) N_{p} * N_{g} \text { (Equation résolvante). }
$$

Si, pour un noyau de convolution $N$ sur $X$, il existe une résolvante $\left(N_{p}\right)_{p>0}$ telle que $\lim _{p \rightarrow 0} N_{p}=N$ (au sens de la topologie vague), alors elle est uniquement déterminée et $\left(N_{p}\right)_{p \geqq 0}$ s'appelle la résolvante associée au noyau $N$, où $N_{0}=N$. Supposons que, pour un noyau de convolution $N$ sur $X$, il existe la résolvante $\left(N_{p}\right)_{p \geqq 0}$ associée au noyau $N$; alors $N$ satisfait au principe complet du maximum si et seulement si, quel que soit $p>0, p \int d N_{p} \leqq 1$.

Proposition 1 (cf. [7]). Soit $N$ un noyau de convolution sur $X$ et supposons qu'il existe la résolvante associée au noyau N. Alors, pour une constante non-négative $c$ et pour une mesure de Radon positive $\nu$ sur $\boldsymbol{R}^{+}$, il existe la résolvante associée au noyau $c \varepsilon+\int N_{p} d \nu(p)$ dès que cela a un sens.

Un noyau de convolution de Hunt $N$ sur $X$ est, par définition, un noyau de convolution sur $X$ de la forme

$$
N=\int_{0}^{\infty} \alpha_{t} d t
$$

où $\left(\alpha_{t}\right)_{t \geqq 0}$ est un semi-groupe vaguement continu de mesures de Radon positives dans $X$; c'est-à-dire, $\alpha_{0}=\varepsilon, \alpha_{t} * \alpha_{s}=\alpha_{t+s} \quad(\forall t \geqq 0, \forall s \geqq 0)$ et l'application $t \rightarrow \alpha_{t}$ est vaguement continue. Dans ce cas, $\left(\alpha_{t}\right)_{t \geqq 0}$ est uniquement déterminé, qui s'appellera le semi-groupe associé au noyau $N$.

Proposition 2 (cf. [11]). Pour qu'un noyau de convolution $N$ sur $X$ soit un noyau de convolution de Hunt sur $X$, il faut et il suffit qu'il 
existe la résolvante associée au noyau $N$ et que $N$ soit non-périodique ${ }^{(1)}$.

Pour un noyau de convolution $N=\int_{0}^{\infty} \alpha_{t} d t$ sur $X$, on obtiendra facilement que les trois énoncés suivants sont équivalents:

(a) $N$ est borné.

(b) $\int d \alpha_{t} \leqq 1(\forall t \geqq 0)$.

(c) Il existe une fonction définie-négative $\psi$ dans le groupe dual $\hat{X}$ de $X$, et une seule telle que la transformée de Fourier de $\alpha_{t}$ soit de la forme $\hat{\alpha}_{t}=\exp (-t \psi)$.

Dans ce cas, $\psi$ s'appelle la fonction définie-négative associée au noyau $N$. On a évidemment $\hat{N}=1 / \psi$ dès que cela a un sens. Une fonction complexe et continue $\psi$ dans $\hat{X}$ est, par définition, définie-négative si l'on a:

(1) $\psi(\hat{O}) \geqq 0$ et $\psi(-\hat{x})=\overline{\psi(\hat{x})}(\forall \hat{x} \in \hat{X})$.

(2) Quels que soient $n$ un entier $\geqq 1,\left(\hat{x}_{i}\right)_{i=1}^{n} \subset \hat{X}$ et $\left(c_{i}\right)_{i=1}^{n}$ nombres complexes avec $\sum_{i=1}^{n} c_{i}=0$,

$$
\sum_{i=1}^{n} \sum_{j=1}^{n} \psi\left(\hat{x}_{i}-\hat{x}_{j}\right) c_{i} \overline{c_{j}} \leqq 0 .
$$

En particular, un noyau de convolution de Hunt symétrique sur $X$ s'appelle un noyau de convolution de Dirichlet sur $X$, qui satisfait toujours au principe complet du maximum.

Proposition 3 (cf. [10] et [11]). Supposons qu'il existe un espace vectoriel topologique $E$ appartenant à $X$ et homéomorphe avec $\boldsymbol{R}$, et soit $\boldsymbol{\kappa}$ un noyau de convolution non-zéro sur $\boldsymbol{R}$ porté par $\boldsymbol{R}^{+}$. Alors pour que, quel que soit $N=\int_{0}^{\infty} \alpha_{t} d t$ un noyau de convolution de Hunt sur $X$, $N_{(\kappa)}=\int \alpha_{t} d \kappa(t)$ soit aussi un noyau de convolution de Hunt sur $X$, il faut et il suffit que $\kappa$ soit un noyau de convolution borné de Hunt sur $\boldsymbol{R}$.

Pour simplifier la notation, $H(A)$ et $H_{b}(A)$ désigneront respectivement la totalité des noyaux de convolution de Hunt sur $X$ portés par $A$ et son sous-ensemble des noyaux de convolution de Hunt bornés, où $A$ est un semi-groupe fermé dans $X$. D'après la proposition 3 , on arrive à la définition suivante:

(1) Cela signifie que, quel que soit $x \neq 0$ de $X, N \neq N * \varepsilon_{x}$, où $\varepsilon_{x}$ est la mesure de Dirac à $x$. 
DÉFinition 1 (cf. [10] et [11]). Soit $N=\int_{0}^{\infty} \alpha_{t} d t$ un noyau de convolution de Hunt sur $X$. On note

$$
H(N ; X)=\left\{N_{(\kappa)}=\int \alpha_{t} d \kappa(t) ; \kappa \in H_{b}\left(\boldsymbol{R}^{+}\right)\right\},
$$

qui s'appelle la famille sous-ordonnée au noyau $N$. On notera ensuite $\bar{H}(N ; X)=H(N ; X) \cup\{0\}$.

Soit $\kappa_{0}$ un noyau d'Heaviside sur $R$; c'est-à-dire $d \kappa_{0}=d t$ sur $\boldsymbol{R}^{+}$et $\kappa_{0}=0$ dans $\boldsymbol{R}-\boldsymbol{R}^{+}$. Alors on a évidemment

$$
H_{b}\left(\boldsymbol{R}^{+}\right)=H\left(\kappa_{0} ; \boldsymbol{R}\right) .
$$

\section{Les cônes convexes de Riesz}

Commençons d'abord avec la définition du principe relatif de domination. Soient $N_{1}$ et $N_{2}$ deux noyaux de convolution sur $X$. On dit que $N_{1}$ satisfait au principe de domination relatif à $N_{2}$ si, quelles que soient $f$ et $g$ de $M_{K}^{+}$, l'inégalité $N_{1} f \leqq N_{2} g$ est satisfaite presque partout pour $\xi$ (noté désormais $\xi$-p.p.) sur $X$ dès qu'elle l'est $\xi$-p.p. sur $\{x \in X$; $f(x)>0\}$. On notera, dans ce cas, $N_{1} \prec N_{2}$. En particulier, si, pour un noyau de convolution $N$ sur $X, N \prec \xi$, on dit qu'il satisfait au principe classique du maximum.

Proposition 4. Soient $N_{0}$ et $H\left(N_{0} ; X\right)$ un noyau de convolution de Hunt sur $X$ et la famille sous-ordonnée au noyau $N_{0}$, respectivement. Alors tout le noyau de convolution $N$ de $H\left(N_{0} ; X\right)$ satisfait au principe de domination relatif $\grave{a} N_{0}$ et $i l$ existe un autre noyau de convolution $N^{\prime}$ sur $X$, et un seul tel que $N * N^{\prime}=N_{0}$.

LEMmE 1. Soit $N=\int_{0}^{\infty} \alpha_{t} d t$ un noyau de convolution de Hunt sur $X$; supposons que, pour trois mesures positives $\lambda_{1}, \lambda_{2}$ et $\lambda_{3}$ dans $\boldsymbol{R}^{+}$, $\lambda_{1} * \lambda_{2}=\lambda_{3}$. Alors $N_{\left(\lambda_{i}\right)}=\int \alpha_{t} d \lambda_{i}(t)(i=1,2)$ a un sens et $a$

$$
N_{\left(\lambda_{1}\right)} * N_{\left(\lambda_{2}\right)}=N_{\left(\lambda_{3}\right)}
$$

dès que $N_{\left(\lambda_{3}\right)}$ a un sens et $\lambda_{3} \neq 0$.

En effet, on a 


$$
\begin{aligned}
N_{\left(\lambda_{3}\right)} & =\int \alpha_{t} d\left(\lambda_{1} * \lambda_{2}\right)(t)=\int \alpha_{t+s} d \lambda_{1}(t) d \lambda_{2}(s) \\
& =\iint \alpha_{t} * \alpha_{s} d \lambda_{1}(t) d \lambda_{2}(s)=N_{\left(\lambda_{1}\right)} * N_{\left(\lambda_{2}\right)},
\end{aligned}
$$

d'où notre lemme.

Lemme 2. Pour un noyau de convolution $\kappa$ de $H_{b}\left(\boldsymbol{R}^{+}\right)$, il existe un autre noyau de convolution $\boldsymbol{\kappa}^{\prime}$ sur $\boldsymbol{R}$ porté par $\boldsymbol{R}^{+}$tel que

$$
d\left(\kappa * \kappa^{\prime}\right)=d t \quad \text { sur } \boldsymbol{R}^{+} \text {et } \quad \frac{d}{d t} \kappa^{\prime} \leqq 0
$$

au sens des distributions dans $(0,+\infty)$.

En effet, soit $\kappa_{0}$ le noyau d'Heaviside sur $\boldsymbol{R}$. Il suffit de montrer que $\kappa$ satisfait au principe de domination relatif à $\kappa_{0}$. Si c'est vrai, il existe un autre noyau de convolution borné $\kappa^{\prime}$ sur $\boldsymbol{R}$ porté par $\boldsymbol{R}^{+}$tel que $\kappa * \kappa^{\prime}=\kappa_{0}$ (cf. [5]). Donc, au sens des distributions, $\left((d / d t) \kappa^{\prime}\right) * \kappa=\varepsilon$, où $\varepsilon$ désigne aussi la mesure de Dirac à l'origine de $\boldsymbol{R}$. $\kappa$ étant de la forme $\kappa=\int_{0}^{\infty} \alpha_{t} d t$, la famille $\left(\left(\varepsilon-\alpha_{t}\right) / t\right)_{t>0}$ converge vers la distribution $(d / d t) \kappa^{\prime}$ au sens des distributions dans $R$ avec $t \downarrow 0$, d'où $(d / d t) \kappa^{\prime} \leqq 0$ dans $(0,+\infty)$. Pour que $\kappa$ satisfasse au principe de domination relatif à $\kappa_{0}$, il suffit que l'implication suivante ait lieu: Quelles que soient $f, g$ de $M_{K}^{+}(R)$,

$$
\kappa f \leqq \kappa_{0} g \text { p.p. } \quad \text { sur } \quad \operatorname{supp}(f d t) \Rightarrow \kappa f \leqq \kappa_{0} g \text { p.p. } \quad \text { sur } \boldsymbol{R}
$$

(cf. [8]). Donc il suffit de voir que, dans tout l'intervalle fermé $[a, b]$ dans le complément de $\operatorname{supp}(f d t), \kappa f \leqq \kappa_{0} g$ p.p. sur $[a, b]$. Soient $f_{a}$ et $g_{a}$ les restreintes de $f$ et de $g \operatorname{sur}(-\infty, a]$; alors $\kappa f=\kappa f_{a}$ et $\kappa_{0} g=\kappa_{0} g_{a}$ sur $(-\infty, b]$. On a

$$
\kappa_{0} g_{a}=\int g_{a} d t \text { p.p. sur }[a, b] \text { et } \kappa_{0} g_{a} \leqq \int g_{a} d t \text { p.p. } \operatorname{sur} \operatorname{supp}\left(f_{a} d t\right) .
$$

$\kappa$ étant borné, il satisfait au principe classique du maximum (cf. par exemple, [9]). Donc $\kappa f_{a} \leqq \int g_{a} d t$ p.p. sur $[a, b]$, d'où $\kappa f \leqq \kappa_{0} g$ p.p. sur $[a, b]$.

On obtiendra facilement l'inverse du lemme 2 dans la section 4. La proposition 4 résultra immédiatement des deux présents lemmes. Soit $\left(\alpha_{t}\right)_{t \geqq 0}$ le semi-groupe associé au noyau $N_{0}$; alors il existe un noyau de 
convolution de Hunt $\kappa$ de $H_{b}\left(\boldsymbol{R}^{+}\right)$tel que $N=\int \alpha_{t} d \kappa(t)$. D'après le lemme 2 , il existe un autre noyau de convolution $\kappa^{\prime}$ sur $\boldsymbol{R}$ tel que $\operatorname{supp}\left(\kappa^{\prime}\right) \subset \boldsymbol{R}^{+}$ et $d\left(\kappa * \kappa^{\prime}\right)(t)=d t$ sur $\boldsymbol{R}^{+}$. D'après le lemme 1 , on a

$$
N * N_{\left(\kappa^{\prime}\right)}=N_{0} \text {. }
$$

Par conséquent, d'après le principe de domination pour $N$, on a évidemment $N \prec N_{0}$.

Pour simplifier la notation, on note $\bar{H}(X)=H(X) \cup\{0\}$ et $\bar{H}_{b}(X)$ $=H_{b}(X) \cup\{0\}$.

DÉFInItion 2. Soit $N_{0}$ un noyau de convolution de Hunt sur $X$. Une famille $C\left(N_{0}\right)$ contenue dans $\bar{H}(X)$ s'appelle un cône convexe de Riesz relatif au noyau $N_{0}$ si elle est un cône convexe vaguement fermé de vertex 0 qui vérifie les conditions suivantes:

(1) $N_{0} \in C\left(N_{0}\right)$.

(2) Pour un noyau de convolution de Hunt quelconque $N$ de $C\left(N_{0}\right)$, il existe un autre noyau de convolution de Hunt $N^{\prime}$ de $C\left(N_{0}\right)$ tel que $N * N^{\prime}=N_{0}$.

Dans (2), $N^{\prime}$ est uniquement déterminé, d'après le principe d'unicité pour $N^{(2)}$, et il s'appellera le noyau dual de $N$ relatif au noyau $N_{0}$. La définition 2 est une notion induite de la formule classique de Riesz et de la proposition 1.

Remarque 1. Soient $N_{0}$ et $C\left(N_{0}\right)$ un noyau de convolution de Hunt sur $X$ et un cône convexe de Riesz relatif au noyau $N_{0}$, respectivement; alors on a, quel que soit $N$ de $C\left(N_{0}\right), N \prec N_{0}$. Cela résulte immédiatement de (2) et du principe de domination pour $N$.

Proposition 5. Soient $N_{0}$ et $C\left(N_{0}\right)$ un noyau de convolution de Hunt sur $X$ et un cône convexe de Riesz relatif au noyau $N_{0}$, respectivement. Alors, pour tout le noyau de convolution de Hunt $N$ de $C\left(N_{0}\right)$, la résolvante associée au noyau $N$ est contenue dans $C\left(N_{0}\right)$.

Démonstration. Soit $N^{\prime}$ le noyau dual de $N$ relatif au noyau $N_{0}$. Alors, quel que soit $p>0, N^{\prime}+p N_{0} \in C\left(N_{0}\right)$. Soit $\tilde{N}_{p}$ le noyau dual de $N^{\prime}+p N_{0}$ relatif au noyau $N_{0}$; alors, quels que soient $p>0$ et $q>0$,

(2) Cela signifie que, quelle que soit $\mu$ une mesure de Radon réelle dans $X, \mu=0$ dès que $N * \mu$ a un sens et $N * \mu=0$. 


$$
\left(N^{\prime}+p N_{0}\right) * \tilde{N}_{p}=\left(N^{\prime}+q N_{0}\right) * \tilde{N}_{q}=N_{0} .
$$

On a, d'après le théorème de Fubini,

$$
\begin{aligned}
\left(N^{\prime}+q N_{0}\right) * \tilde{N}_{q} & =\left(N^{\prime}+p N_{0}+(q-p)\left(N^{\prime}+p N_{0}\right) * \tilde{N}_{p}\right) * \tilde{N}_{q} \\
& =\left(N^{\prime}+p N_{0}\right) *\left(\tilde{N}_{q}+(q-p) \tilde{N}_{p} * \tilde{N}_{q}\right) .
\end{aligned}
$$

$N^{\prime}+p N_{0}$ satisfaisant au principe d'unicité, on a $\tilde{N}_{p}-\tilde{N}_{q}=(q-p) \tilde{N}_{p} * \tilde{N}_{q}$, et donc $\left(\tilde{N}_{p}\right)_{p>0}$ est une résolvante. On a aussi $N-\tilde{N}_{p}=p N * \tilde{N}_{p}\left({ }^{\forall} p>0\right)$. $N$ étant un noyau de convolution de Hunt sur $X$, on a $\lim _{p \rightarrow 0} N_{p}=N$ (au sens de la topologie vague), d'où $\left(\tilde{N}_{p}\right)_{p \geqq 0}$ est la résolvante associée au noyau $N$, où $\tilde{N}_{0}=N$. La démonstration est ainsi complète.

On notera $m_{1}\left(\boldsymbol{R}^{+}\right)$et $m(X)$ la totalité des mesures de Radon positives dans $\boldsymbol{R}^{+}$de masse totale finie et la totalité des mesures de Rodon positives dans $X$, respectivement.

DÉFINITION 3. Soit $\left(\alpha_{t}\right)_{t \geqq 0}$ un semi-groupe vaguement continu de mesures de Radon positives dans $X$ tel que, quelle que soit $\nu$ de $m_{1}\left(\boldsymbol{R}^{+}\right)$, $\int \alpha_{t} d \nu(t)$ a un sens dans $m(X)$. Si l'application $m_{1}\left(\boldsymbol{R}^{+}\right) \ni \nu \rightarrow \int \alpha_{t} d \nu(t) \in m(X)$ est injective, alors ce semi-groupe est dit injectif.

Pour un noyau de convolution de Hunt $N=\int_{0}^{\infty} \alpha_{t} d t \operatorname{sur} X, \int \alpha_{t} d \nu(t)$ a un sens dans $m(X)\left(\forall_{\nu} \in m_{1}\left(\boldsymbol{R}^{+}\right)\right)$.

Remarque 2. Soit $\left(\alpha_{t}\right)_{t \geqq 0}$ un semi-groupe vaguement continu de mesures de Radon positives dans $X$ tel que $\int d \alpha_{t} \leqq 1(t \geqq 0)$; alors il existe une fonction définie-négative $\psi$ dans $\hat{X}$, et une seule telle que l'on ait $\hat{\alpha}_{t}=\exp (-t \psi) \quad(t \geqq 0) \quad$ (cf. par exemple, [4]). Si $\{\psi(\hat{x}) \in C ; \hat{x} \in \hat{X}\}$ $\cap\{z \in C$; Re. $z>0\}$ contient un ensemble relativement compact et infini dans $\{z \in C$; Re. $z>0\}$, alors $\left(\alpha_{t}\right)_{t \geqq 0}$ est injectif. On note ici $C$ le champ complexe et Re. $z$ désigne la partie réelle de $z$.

En effet, il est évident que, quelle que soit $\nu$ de $m_{1}\left(\boldsymbol{R}^{+}\right), \int \alpha_{t} d \nu(t)$ a un sens dans $m(X)$, et on a

$$
\widehat{\int \alpha_{t} d \nu}(t)(\hat{x})=\int \exp (-t \psi(\hat{x})) d \nu(t)
$$

pour tout $\hat{x}$ de $\hat{X} . \quad$ Posons $F_{\nu}(z)=\int \exp (-t z) d \nu(t)$ sur $\{z \in C ;$ Re. $z \geqq 0\}$; 
alors $F_{\nu}$ est continue sur $\{z \in C$; Re. $z \geqq 0\}$ et elle est analytique dans $\{z \in C ; \operatorname{Re} . z>0\}$. En utilisant le théorème de coïncidence dans la théorie classique de fonction et le fait que la transformation de Laplace est injective, on peut affirmer facilement que $\left(\alpha_{t}\right)_{t \geqq 0}$ est injectif.

Rappelons ensuite le théorème de Bernstein dans la théorie classique d'analyse harmonique.

Proposition 4 (cf. par exemple, [13]). Soit $\varphi$ une fonction nonnégative et infiniment dérivable dans $(0,+\infty)$. Pour que $\varphi$ soit complètement monotone, il faut et il suffit qu'il existe une measure de Radon positive $\lambda$ sur $\boldsymbol{R}^{+}$telle que

$$
\varphi(t)=\int \exp (-t s) d \lambda(s) \text { dans }(0,+\infty)
$$

On dit que $\varphi$ est complètement monotone si, quel que soit $m$ un entier non-négatif, $(-1)^{m} \varphi^{(m)}(t) \geqq 0$ dans $(0,+\infty)$, où $\varphi^{(0)}=\varphi$ et $\varphi^{(m)}$ est la dérivée de $\varphi$ d'ordre $m$.

Nous montrerons notre premier théorème principal suivant:

THÉORÈME 1. Soient $N$ et $\left(N_{p}\right)_{p \geqq 0}$ respectivement un noyau de convolution de Hunt sur $X$ et la résolvante associée au noyau $N$. Alors la totalité des noyaux de convolution sur $X$ de la forme $c \varepsilon+\int N_{p} d \lambda(p)$ est un cône convexe de Riesz relatif au noyau $N$, où c et $\lambda$ sont une constante non-négative et une mesure de Radon positive dans $\boldsymbol{R}^{+}$, respectivement. En particulier, si le semi-groupe associé au noyau $N$ est injective, alors cela est un seul cône convexe de Riesz relatif au noyau $N$ appartenant à $\bar{H}(N ; X)$.

On préparera d'abord un lemme élémentaire.

LEMME 3. Soient $n,\left(p_{i}\right)_{i=0}^{n},\left(a_{i}\right)_{i=0}^{n}$ et $c$ un entier positif, une famille de nombres avec $0=p_{0}<p_{1}<\cdots<p_{n}$, une famille de nombres positifs et un nombre positif, respectivement. La fonction

$$
f(t)=\frac{1}{c t+\sum_{i=0}^{n} \frac{a_{i} t}{t+p_{i}}}
$$

sur $\boldsymbol{R}$ est de la forme 


$$
f(t)=\sum_{i=0}^{n} \frac{b_{i}}{t+q_{i}}
$$

où $p_{i}<q_{i}<p_{i+1}(i=0,1, \cdots, n-1), q_{n}>p_{n}$ et $b_{i}>0(i=0,1, \cdots, n)$.

Posons

$$
g(t)=c t\left(t+p_{1}\right) \cdots\left(t+p_{n}\right)+\sum_{i=0}^{n} \frac{a_{i} t\left(t+p_{1}\right) \cdots\left(t+p_{n}\right)}{t+p_{i}}
$$

alors $g\left(-p_{2 k}\right)>0$ et $g\left(-p_{2 m+1}\right)<0$ dès que $2 k \leqq n$ et $2 m+1 \leqq n$. D'autre part, il existe une constante positive $A$ telle que, quel que soit $t \geqq A$, $(-1)^{n} g(-t)<0$. Donc il existe une famille $\left(q_{i}\right)_{i=0}^{n}$ de nombres positifs telle que $g\left(-q_{i}\right)=0(0 \leqq i \leqq n), p_{i}<q_{i}<p_{i+1}(0 \leqq i \leqq n-1)$ et $q_{n}>p_{n}$. Donc on peut écrire

$$
f(t)=\sum_{i=0}^{n} \frac{b_{i}}{t+q_{i}},
$$

où $b_{i}$ est une constante réelle. Remarquons

$$
f(t)=\frac{\left(t+p_{1}\right) \cdots\left(t+p_{n}\right)}{g(t)}
$$

on a alors

$$
\lim _{t \downarrow-q_{i}} f(t)=+\infty
$$

et par suite $b_{i}>0(0 \leqq i \leqq n)$, d'où notre lemme.

Démonstration du théorème 1 . Soit $\kappa_{0}$ le noyau d'Heaviside sur $\boldsymbol{R}$; $\left(\kappa_{p}\right)_{p \geqq 0}$ désigne la résolvante associée au noyau $\kappa_{0}$. On a alors $\kappa_{p}=0$ dans $(-\infty, 0)$ et $d \kappa_{p}(t)=\exp (-p t) d t \operatorname{sur} \boldsymbol{R}^{+}$. On notera $C_{R}\left(\kappa_{0}\right)$ la totalité des noyaux de convolution sur $\boldsymbol{R}$ de la forme $c \varepsilon+\int \kappa_{p} d \lambda(p)$, où $c$ et $\lambda$ sont une constante non-négative et une mesure de Radon positive sur $\boldsymbol{R}^{+}$, respectivement.

On montrera d'abord que $C_{R}\left(\kappa_{0}\right)$ est un seul cône convexe de Riesz relatif au noyau $\kappa_{0}$. Il est bien connu que $C_{R}\left(\kappa_{0}\right)$ appartient à $\bar{H}\left(\kappa_{0} ; \boldsymbol{R}\right)$ $=\bar{H}_{b}\left(\boldsymbol{R}^{+}\right)$(cf. [7] et [11]). Evidemment $C_{R}\left(\kappa_{0}\right)$ est un cône convexe de vertex 0 . D'après le lemme 1 , pour un noyau de convolution de Hunt $c \varepsilon+\int \kappa_{p} d \lambda(p)$ de $C_{R}\left(\kappa_{0}\right)$, il existe un noyau de convolution $\kappa^{\prime}$ sur $\boldsymbol{R}$ porté 
par $\boldsymbol{R}^{+}$, et un seul tel que l'on ait

$$
\left(c \varepsilon+\int \kappa_{p} d \lambda(p)\right) * \kappa^{\prime}=\kappa_{0} .
$$

Pour que $\kappa^{\prime}$ appartienne à $C_{R}\left(\kappa_{0}\right)$, il suffit que, quel que soit $m$ un entier non-négatif, $(-1)^{m}\left(d^{m} / d t^{m}\right) \kappa^{\prime} \geqq 0$ au sens des distributions dans $(0,+\infty)$, d'après le théorème de Bernstein. Soit $\kappa_{n}^{\prime}$ un noyau de convolution sur $X$ qui vérifie l'égalité

$$
\left(\left(c+\frac{1}{n}\right) \varepsilon+\int_{0}^{n} \kappa_{p} d \lambda(p)\right) * \kappa_{n}^{\prime}=\kappa_{0}
$$

alors il est uniquement déterminé, et la suite $\left(\kappa_{n}^{\prime}\right)_{n=1}^{\infty}$ converge vaguement vers $\kappa^{\prime}$ avec $n \rightarrow+\infty$. Donc on peut supposer que $c>0$ et $\lambda$ est à support compact. Si $\lambda$ est de la forme

$$
\lambda=\sum_{i=0}^{n} a_{i} \varepsilon_{p_{i}},
$$

où $a_{i}>0, p_{0}=0, p_{i}>0(1 \leqq i \leqq n)$ et $\varepsilon_{p_{i}}$ est la mesure de Dirac au point $p_{i}$, on a alors

$$
\left(c+\sum_{i=1}^{n} \frac{a_{i}}{t+p_{i}}\right) \widetilde{\kappa^{\prime}}(t)=\frac{1}{t}
$$

dans $(0,+\infty)$, où $\widetilde{\kappa^{\prime}}$ est la transformée de Laplace de $\kappa^{\prime}$. On remarque ici que $\widetilde{\kappa^{\prime}}$ a un sens, d'après le fait que $\kappa^{\prime}$ est borné (cf. le lemme 2). Donc

$$
\widetilde{\kappa^{\prime}}(t)=\frac{1}{c t+\sum_{i=0}^{n} \frac{a_{i} t}{t+p_{i}}}
$$

D'après le lemme 3 , on a

$$
\widetilde{\kappa^{\prime}}(t)=\sum_{i=0}^{\infty} \frac{b_{i}}{t+q_{i}}
$$

où $q_{i}>0$ et $b_{i}>0$, et par suite

$$
\kappa^{\prime}=\sum_{i=0}^{n} b_{i} \kappa_{q_{i}}
$$

d'où, quel que soit $m$ un entier non-négatif, $(-1)^{m}\left(d^{m} / d t^{m}\right) \kappa^{\prime} \geqq 0$ au sens 
des distributions dans $(0,+\infty)$. En général, pour une mesure de Radon positive $\lambda$ dans $\boldsymbol{R}^{+}$à support compact, il existe une suite $\left(\lambda_{n}\right)_{n=1}^{\infty}$ de mesures de Radon positives sur $\boldsymbol{R}^{+}$de la présente forme qui converge vaguement vers $\lambda$ avec $n \rightarrow+\infty$. On note encore $\kappa_{n}^{\prime}$ le noyau de convolution sur $\boldsymbol{R}$ tel que

$$
\left(c \varepsilon+\int \kappa_{p} d \lambda_{n}(p)\right) * \kappa_{n}^{\prime}=\kappa_{0}
$$

et alors la suite $\left(\kappa_{n}^{\prime}\right)_{n=1}^{\infty}$ converge vaguement vers $\kappa^{\prime}$ avec $n \rightarrow+\infty$. On a donc, quel que soit $m$ un entier non-négatif, $(-1)^{m}\left(d^{m} / d t^{m}\right) \kappa^{\prime} \geqq 0$ au sens des distributions dans $(0,+\infty)$, d'où $\kappa^{\prime} \in C_{R}\left(\kappa_{0}\right)$. Supposons qu'une suite de $C_{R}\left(\kappa_{0}\right)$ converge vaguement vers un noyau de convolution $\kappa$ sur $\boldsymbol{R}$; alors on a aussi $(-1)^{m}\left(d^{m} / d t^{m}\right) \kappa \geqq 0$ au sens des distribution dans $(0,+\infty)$, et donc, d'après la proposition $6, \kappa$ appartient à $C_{R}\left(\kappa_{0}\right)$. Par conséquent, $C_{R}\left(\kappa_{0}\right)$ est vaguement fermé, d'où $C_{R}\left(\kappa_{0}\right)$ est un cône convexe de Riesz relatif au noyau $\kappa_{0}$.

Soit $C\left(\kappa_{0}\right)$ un autre cône convexe de Riesz relatif au noyau $\kappa_{0}$. D'après la proposition 5 , on a $C_{R}\left(\kappa_{0}\right) \subset C\left(\kappa_{0}\right)$. On remarque ici que $\varepsilon \in C\left(\kappa_{0}\right)$ résulte de $\kappa_{0} \in C\left(\kappa_{0}\right)$. D'après le lemme 2 , on a, quel que soit $\kappa$ de $C\left(\kappa_{0}\right),-(d / d t)_{\kappa} \geqq 0$ au sens des distributions dans $(0,+\infty)$. Soit $m$ un entier positif et supposons que, quel que soit $\kappa$ de $C\left(\kappa_{0}\right),(-1)^{m}\left(d^{m} / d t^{m}\right) \kappa \geqq 0$ au sens des distributions dans $(0,+\infty)$. Soit $\kappa$ un noyau de convolution de Hunt quelconque de $C\left(\kappa_{0}\right) ; \kappa^{\prime}$ désigne le noyau dual de $\kappa$ relatif au noyau $\kappa_{0}$. On note ensuite $\left(\kappa_{p}^{\prime}\right)_{p \geqq 0}$ la résolvante associée au noyau $\kappa^{\prime}$; alors $\left(\kappa_{p}^{\prime}\right)_{p \geqq 0} \subset C\left(\kappa_{0}\right)$ (cf. la proposition 5). On a

$$
\left(p \kappa_{p}^{\prime}\right) * \kappa=p \kappa * \kappa^{\prime}-p^{2} \kappa * \kappa^{\prime} * \kappa_{p}^{\prime}=p \kappa_{0}-p^{2} \kappa_{0} * \kappa_{p}^{\prime},
$$

et par suite

$$
(-1)^{m+1} \frac{d^{m+1}}{d t^{m+1}}\left(p \kappa_{p}^{\prime}\right) * \kappa=p(-1)^{m+1} \frac{d^{m}}{d t^{m}} \varepsilon+p^{2}(-1)^{m} \frac{d^{m}}{d t^{m}} \kappa_{p}^{\prime}
$$

Par conséquent, on a

$$
(-1)^{m+1} \frac{d^{m+1}}{d t^{m+1}}\left(p \kappa_{p}^{\prime}\right) * \kappa \geqq 0
$$

au sens des distributions dans $(0,+\infty)$. Faisant $p \rightarrow+\infty$, on obtient 


$$
(-1)^{m+1} \frac{d^{m+1}}{d t^{m+1}} \kappa \geqq 0
$$

au sens des distributions dans $(0,+\infty)$, car $\lim _{p \rightarrow+\infty} p \kappa_{p}^{\prime}=\varepsilon$ (au sens de la topologie vague). Par récurrence on obtient, quels que soient $\kappa$ de $C\left(\kappa_{0}\right)$ et $m$ un entier non-négatif, $(-1)^{m}\left(d^{m} / d t^{m}\right) \kappa \geqq 0$ au sens des distributions dans $(0,+\infty)$, d'où $C_{R}\left(\kappa_{0}\right) \supset C\left(\kappa_{0}\right)$. Donc $C_{R}\left(\kappa_{0}\right)$ est un seul cône convexe de Riesz relatif au noyau $\kappa_{0}$.

Soient $N$ un noyau de convolution de Hunt sur $X$ et $\left(N_{p}\right)_{p \geqq 0}$ la résolvante associée au noyau $N$. On désignera par $C_{R}(N)$ la totalité des noyaux de convolution sur $X$ de la forme $c \varepsilon+\int N_{p} d \lambda(p)$, où $c$ et $\lambda$ sont respectivement une constante non-négative et une mesure de Radon positive dans $\boldsymbol{R}^{+}$. Alors cela est évidemment un cône convexe de vertex 0 vérifiant les conditions (1), (2) dans la définition 1 , car on a, quelle que soit $\lambda$ une mesure de Radon positive sur $\boldsymbol{R}^{+}$,

$$
\int N_{p} d \lambda(p)=\int_{0}^{\infty} \alpha_{t} \int \exp (-p t) d \lambda(p) d t
$$

où $\left(\alpha_{t}\right)_{t \geqq 0}$ est le semi-groupe associé au noyau $N$. Montrons que $C_{R}(N)$ est vaguement fermé. Si une famille $\left(\int N_{p} d \lambda_{\alpha}(p)\right)_{\alpha \in \Lambda}$ est vaguement bornée dans $X$, alors $\left(\lambda_{\alpha}\right)_{\alpha \in \Lambda}$ est aussi vaguement bornée sur $\boldsymbol{R}^{+}$. Donc il suffit de montrer que si une suite $\left(\lambda_{n}\right)_{n=1}^{\infty}$ de mesures de Radon positives sur $\boldsymbol{R}^{+}$ converge vaguement vers une mesure de Radon positive $\lambda$ sur $\boldsymbol{R}^{+}$et si $\left(\int N_{p} d \lambda_{n}(p)\right)_{n=1}^{\infty}$ est vaguement bornée dans $X$, alors tout sa sous-suite converge vaguement vers un noyau de convolution de $C_{R}(N)$ dès qu'elle converge vaguement. On a, quelle que soit $f$ une fonction de $C_{K}(R)$,

$$
\lim _{n \rightarrow+\infty} \int f(p) N_{p} d \lambda_{n}(p)=\int f(p) N_{p} d \lambda(p)
$$

au sens de la topologie vague dans $X$. Donc on peut supposer qu'il existe un nombre $r>0$ tel que $\operatorname{supp}\left(\lambda_{n}\right) \subset[r,+\infty)\left({ }^{\forall} n \geqq 1\right)$. Evidemment la suite $\left(\int \kappa_{p} d \lambda_{n}(p)\right)_{n=1}^{\infty}$ est vaguement bornée dans $R$, et on peut supposer qu'elle converge vaguement vers un noyau de convolution $\kappa$ de $C_{R}\left(\kappa_{0}\right)$ avec $n \rightarrow+\infty$. De la même manière que pour $C_{R}\left(\kappa_{0}\right)$, on obtient que $C_{R}\left(\kappa_{r}\right)$ est aussi un cône convexe de Riesz relatif au noyau $\kappa_{r}$. La famille 
$\left(\kappa_{r+p}\right)_{p \geqq 0}$ étant la résolvante associée au noyau $\kappa_{r}$, on a $\left(\int \kappa_{p} d \lambda_{n}(p)\right)_{n=1}^{\infty}$ $\subset C_{R}\left(\kappa_{r}\right)$ et $\kappa \in C_{R}\left(\kappa_{r}\right)$. On peut supposer $\lambda_{n} \neq 0(\forall n \geqq 1), \lambda \neq 0$, et donc $\kappa \neq 0$. Soient $\kappa_{n}^{\prime}$ et $\kappa^{\prime}$ le noyau dual de $\int \kappa_{p} d \lambda_{n}(p)$ et le noyau dual de $\kappa$ relatifs au noyau $\kappa_{r}$, respectivement. Alors $\left(\kappa_{n}^{\prime}\right)_{n=1}^{\infty}$ converge vaguement vers $\kappa^{\prime}$ avec $n \rightarrow+\infty$. Ayant

$$
\iint d \kappa_{p} d \lambda_{n}(p) \int d \kappa_{n}^{\prime}=\int \frac{1}{p} d \lambda_{n}(p) \int d \kappa_{n}^{\prime}=\int d \kappa_{r}=\frac{1}{r} \text { et } \int d \kappa \int d \kappa^{\prime}=\frac{1}{r},
$$

on obtient que la suite $\left(\int \frac{1}{p} d \lambda_{n}(p)\right)_{n=1}^{\infty}$ est bornée. D'autre part, on a, quelle que soit $f$ une fonction de $C_{K}(X), \lim _{t \rightarrow+\infty} \alpha_{t} * f(0)=0$. Donc

$$
\lim _{n \rightarrow+\infty} \int N_{p} d \lambda_{n}(p)=\int \alpha_{t} d \kappa(t)
$$

au sens de la topologie vague dans $X$. Par conséquent, $C_{R}(N)$ est vaguement fermé, d'où $C_{R}(N)$ est un cône convexe de Riesz relatif au noyau $N$.

Supposons finalement que le semi-groupe $\left(\alpha_{t}\right)_{t \geq 0}$ associé au noyau $N$ est injectif. Soit $C(N)$ un cône convexe de Riesz quelconque relatif au noyau $N$ appartenant à $\bar{H}(N ; X)$. Pour que $C(N)=C_{R}(N)$, il suffit de montrer que la famille

$$
C_{R}^{\prime}\left(\kappa_{0}\right)=\left\{\kappa \in \bar{H}_{b}\left(R^{+}\right) ; \int \alpha_{t} d \kappa(t) \in C(N)\right\}
$$

appartient à $C_{R}\left(\kappa_{0}\right)$, car on a toujours $C(N) \supset C_{R}(N) . \quad C_{R}^{\prime}\left(\kappa_{0}\right)$ est évidemment un cône convexe de vertex 0 . Soit $\kappa$ un noyau de convolution de $H_{b}\left(\boldsymbol{R}^{+}\right) \cap C_{R}^{\prime}\left(\kappa_{0}\right)$; alors il existe un autre noyau de convolution $\kappa^{\prime}$ de $H_{b}\left(\boldsymbol{R}^{+}\right) \cap C_{R}^{\prime}\left(\kappa_{0}\right)$ tel que

$$
N=\left(\int \alpha_{t} d \kappa(t)\right) *\left(\int \alpha_{t} d \kappa^{\prime}(t)\right)=\int \alpha_{t} d\left(\kappa * \kappa^{\prime}\right)(t)
$$

Soient $\left(\tilde{\kappa}_{p}\right)_{p \geq 0}$ et $\left(\tilde{\kappa}_{p}^{\prime}\right)_{p \geqq 0}$ respectivement la résolvante associée au noyau $\kappa$ et la résolvante associée au noyan $\tilde{\kappa}$; on a alors, pour trois nombres $p>0, q>0$ et $r>0$,

$$
\int \alpha_{t} d \kappa_{p} *\left(\varepsilon-q \tilde{\kappa}_{q}\right) *\left(\varepsilon-r \tilde{\kappa}_{r}^{\prime}\right)(t)=\int \alpha_{t} d \tilde{\kappa}_{q} * \tilde{\kappa}_{r}^{\prime} *\left(\varepsilon-p \kappa_{p}\right)(t) .
$$


Ayant $\int d\left|\kappa_{p} *\left(\varepsilon-p \tilde{\kappa}_{p}\right) *\left(\varepsilon-p \tilde{\kappa}_{p}^{\prime}\right)\right|<+\infty$ et $\int d\left|\tilde{\kappa}_{q} * \tilde{\kappa}_{r}^{\prime} *\left(\varepsilon-p \kappa_{p}\right)\right|<+\infty$, on obtient

$$
\kappa_{p} *\left(\varepsilon-q \tilde{\kappa}_{q}\right) *\left(\varepsilon-r \kappa_{r}^{\prime}\right)=\tilde{\kappa}_{q} * \tilde{\kappa}_{r}^{\prime} *\left(\varepsilon-p \kappa_{p}\right) .
$$

Faisant séparément $p \downarrow 0, q \downarrow 0$ et $r \downarrow 0$, on a $\kappa_{0}=\kappa * \kappa^{\prime}$. Donc, de la même manière que ci-dessus, on a $C_{R}^{\prime}\left(\kappa_{0}\right) \subset C_{R}\left(\kappa_{0}\right)$. La démonstration est ainsi complète.

Remarque 3. Soient $N$ un noyau de convolution de Hunt sur $X$ et $\left(C_{\alpha}(N)\right)_{\alpha \in \Lambda}$ une famille de cônes convexes de Riesz relatifs au noyau $N$. Alors $\bigcap_{\alpha \in A} C_{\alpha}(N)$ est aussi un cône convexe de Riesz relatif au noyau $N$. $C_{R}(N)$ est le plus petit cône convexe de Riesz relatif au noyau $N$.

\section{Les noyaux de convolution complètement sous-harmoniques en dehors de l'origine.}

Dès maintenant, $X$ est toujours l'espace euclidien $\boldsymbol{R}^{n}$ à $n(\geqq 1)$ dimensions. Pour un point $x=\left(x_{1}, x_{2}, \cdots, x_{n}\right)$ de $\boldsymbol{R}^{n}$, on notera $|x|$ $=\left(x_{1}^{2}+x_{2}^{2}+\cdots+x_{n}^{2}\right)^{1 / 2}$. On désignera par $\mathscr{D}=\mathscr{D}\left(\boldsymbol{R}^{n}\right)$ et $\mathscr{S}=\mathscr{S}\left(\boldsymbol{R}^{n}\right)$ l'espace vectoriel topologique usuel des fonctions complexes et infiniment dérivables dans $X$ à support compact, et l'espace vectoriel topologique usuel des fonctions complexes et infiniment dérivables dans $X$ à décroissance rapide. Soit $k$ un entier non-négatif. On notera

$\theta_{0}^{k}\left(\right.$ resp. $\left.\theta_{s}^{k}\right)$ l'idéal de $\mathscr{D}$ (resp. $\mathscr{S}$ ) constitué par toutes les fonctions qui s'annulent ainsi que leurs dérivées d'ordre $<k$ à l'origine,

$\widehat{\theta}_{0}^{k}$ (resp. $\widehat{\theta}_{s}^{k}$ ) l'idéal de convolution formé des fonctions $\varphi \in \mathscr{D}$ (resp. $\in \mathscr{S})$ telles que, quel que soit $\alpha$ un multi-indice avec $|\alpha|<k, \int x^{\alpha} \varphi(x) d x=0$.

Pour un multi-indice $\alpha=\left(\alpha_{1}, \alpha_{2}, \cdots, \alpha_{n}\right)$, on écrit $|\alpha|=\alpha_{1}+\alpha_{2}+\cdots$ $+\alpha_{n}$. Pour $x=\left(x_{1}, x_{2}, \cdots, x_{n}\right)$ de $\boldsymbol{R}^{n}, x^{\alpha}$ désigne $x_{1}^{\alpha_{1}} x_{2}^{\alpha_{2}} \cdots x_{n}^{\alpha_{n}}$. La définition suivante est mis en ordre par C. S. Herz [4].

DÉFINITION 4. (i) Soit $k$ un entier non-négatif. Une distribution $u$ dans $R^{n}$ sera dite $k$-conditionnellement positive si, quelle que soit $\varphi$ de $\theta_{0}^{k}, u\left(|\varphi|^{2}\right) \geqq 0$.

(ii) Une distribution $v$ dans $\boldsymbol{R}^{n}$ sera dite $k$-conditionnellement de type positif si, quelle que soit $\varphi$ de $\widehat{\theta_{0}^{k}}, v * \varphi * \stackrel{*}{\varphi}(0) \geqq 0$, où $\stackrel{*}{\varphi}(x)=\overline{\varphi(-x)}$.

Si une distribution $v$ dans $\boldsymbol{R}^{n}$ est $k$-conditionnellement de type positif, 
alors $v$ est une distribution dans $\boldsymbol{R}^{n}$ à croissance lente (cf. [4]), et donc, quelle que soit $\varphi$ de $\theta_{s}^{k}, v * \varphi * \stackrel{*}{\varphi}(0) \geqq 0$, car $\theta_{0}^{k}$ est dense dans $\theta_{s}^{k}$. C. S. Herz [4] montre le théorème fondamental suivant, qui est une généralisation $\mathrm{du}$ théorème de Bochner.

Proposition 7. Les deux énoncés suivants sont équivalents:

(a) Une distribution $v$ dans $\boldsymbol{R}^{n}$ est k-conditionnellement de type positif.

(b) $v=\hat{u}$, ò̀ $u$ est une distribution $k$-conditionnellement positive dans $\boldsymbol{R}^{n}$ à croissance lente.

Rappelons ici que, dans cette note, la signe $\wedge$ représente la transformation de Fourier. D'après la définition de fonction définie-négative, on obtient immédiatement que, pour une fonction définie-négative $\psi$ dans $\boldsymbol{R}^{n},-\psi$ est 1-conditionnellement de type positif. Rappelons que, d'après le théorème de Lévy-Khinchine, une fonction définie-négative $\psi$ dans $\boldsymbol{R}^{n}$ est de la forme

$$
\begin{aligned}
\psi(x)= & c+\sum_{i=1}^{n} \sum_{j=1}^{n} a_{i j} x_{i} x_{j}+\sum_{i=1}^{n} b_{i} x_{i} \sqrt{-1} \\
& +\int\left(1-\frac{x \cdot y}{1+|y|^{2}} \sqrt{-1}-\exp (x \cdot y \sqrt{-1})\right) d \sigma(y),
\end{aligned}
$$

où $x=\left(x_{1}, x_{2}, \cdots, x_{n}\right), y=\left(y_{1}, y_{2}, \cdots, y_{n}\right), x \cdot y=\sum_{i=1}^{n} x_{i} y_{i} ; c \geqq 0, a_{i j}$ et $b_{i}(i, j=1,2, \cdots, n)$ sont constantes réelles; $\sum_{i=1}^{n} \sum_{j=1}^{n} a_{i j} x_{i} x_{j}$ est une forme quadratique non-négative; $\sigma$ est une mesure de Radon positive en dehors de l'origine avec

$$
\int \frac{|x|^{2}}{1+|x|^{2}} d \sigma(x)<+\infty .
$$

Remarque 4. Soient $\psi$ une fonction définie-négative dans $\boldsymbol{R}^{n}$ et $\left(\alpha_{t}\right)_{t \geqq 0}$ le semi-groupe vaguement continu de mesures de Radon positives dans $\boldsymbol{R}^{n}$ tel que $\hat{\alpha}_{t}=\exp (-t \psi)$. Alors $\left(\alpha_{t}\right)_{t \geqq 0}$ est injectif dès que $\psi \neq 0$. Cela résulte immédiatement de la remarque 2 .

En particulier, si une fonction définie-négative $\psi$ dans $\boldsymbol{R}^{n}$ est invariante par rotations, alors elle est non-négative et de la forme

$$
\psi(x)=c_{1}+c_{2}|x|^{2}+\int(1-\exp (x \cdot y \sqrt{-1})) d \sigma(y)
$$


où $c_{i}(i=1,2)$ est une constante non-négative et $\sigma$ est une mesure de Radon positive en dehors de l'origine invariante par rotations et vérifiant la présente inégalité.

LEMmE 4. Soit $\varphi$ une fonction non-négative et continue sur $\boldsymbol{R}^{+}$. Si, pour un entier positif $m, \psi_{m}(x)=\varphi(|x|)$ est définie-négative dans $\boldsymbol{R}^{m}$, alors, quel que soit $n$ un entier positif avec $n \leqq m$, la fonction $\psi_{n}(x)$ $=\varphi(|x|)$ dans $\boldsymbol{R}^{n}$ est aussi définie-négative.

Il est facile de voir ce lemme. Dans le présent lemme, pour un entier $n>m$, la fonction $\psi_{n}(x)=\varphi(|x|)$ dans $\boldsymbol{R}^{n}$ n'est pas toujours définienégative (voir [11]).

DÉFINITION 5. (i) Un noyau de convolution $N$ sur $\boldsymbol{R}^{n}$ est conditionnellement sous-harmonique si l'on a $\Delta N \geqq 0$ au sens des distributions en dehors de l'origine.

(ii) $N$ est dit complètement sous-harmonique en dehors de l'origine si l'on a, quel que soit $k$ un entier non-négatif, $\Delta^{k} N \geqq 0$ au sens des distributions en dehors de l'origine.

On note ici $\Delta$ l'opérateur de Laplace sur $R^{n}$, et $\Delta^{1}=\Delta, \Delta^{k}=\left(\Delta^{k-1}\right) \Delta$.

LEMme 5. Soit $\varphi$ une fonction infiniment dérivable dans $(0,+\infty)$. Si, quel que soit $k$ un entier non-négatif, $\varphi^{(2 k)} \geqq 0$ et $\varlimsup_{\lim _{t \rightarrow+\infty}} \varphi(t)<+\infty$, alors $\varphi$ est complètement monotone.

En effet, $\varphi$ est convexe et on a $\varlimsup_{t \rightarrow+\infty} \varphi(t)<+\infty$, et donc $-\varphi^{\prime}(t) \geqq 0$ dans $(0,+\infty)$. Supposons qu'il existe un entier positif $k$ tel que $\varphi^{(2 k)}$ ne soit pas décroissante au sens large. On peut supposer ici que, quel que soit $m$ un entier non-négatif avec $m<k, \varphi^{(2 m)}$ est décroissante au sens large. Donc $\lim _{t \rightarrow+\infty} \varphi^{(2 m)}(t)<+\infty$. Soit $t_{0}$ un nombre positif tel que $\varphi^{(2 k+1)}\left(t_{0}\right)>0$. Alors

$$
\varphi^{(2 k+1)}(t) \geqq \varphi^{(2 k+1)}\left(t_{0}\right) \quad \text { sur } \quad\left[t_{0} ; \infty\right),
$$

et donc

$$
\varphi^{(2 k)}(t) \geqq \varphi^{(2 k+1)}\left(t_{0}\right)\left(t-t_{0}\right)
$$

pour tout $t$ de $\left[t_{0},+\infty\right)$. Soit $f$ une fonction non-négative et non-zéro de $\mathscr{D}\left(\boldsymbol{R}^{1}\right)$; alors

$$
\varliminf_{t \rightarrow+\infty} \int \varphi^{(2 k)}(|t-s|) f(s) d s \geqq \varliminf_{t \rightarrow+\infty} \int \varphi^{(2 k+1)}\left(t_{0}\right)\left(|t-s|-t_{0}\right) f(s) d s=+\infty .
$$


On a, d'autre part,

$$
\lim _{t \rightarrow+\infty} \int \varphi^{(2 k)}(|t-s|) f(s) d s=\lim _{t \rightarrow+\infty} \int \varphi^{(2 k-2)}(|t-s|) f^{\prime \prime}(s) d s<+\infty,
$$

d'où une contradiction. Par conséquent, quel que soit $m$ un entier nonnégatif, $(-1)^{m} \varphi^{(m)} \geqq 0$.

Dans le présent lemme, on peut remplacer $\lim _{t \rightarrow+\infty} \varphi(t) / t=0$ au lieu de la condition $\varlimsup_{\lim },+(t)<+\infty$. Dès maintenant, nous considérerons principalement les noyaux de convolution sur $\boldsymbol{R}^{n}$ invariants par rotations.

LEMME 6. Soit $N$ un noyau de convolution borné et conditionnellement sous-harmonique sur $\boldsymbol{R}^{n}$ invariant par rotations; alors il existe une fonction définie-négative $\psi$ dans $\boldsymbol{R}^{n}$ invariante par rotations, et une seule telle que $\psi=-\widehat{\Delta N}$.

En effet, on remarque d'abord que $N$ s'écrit

$$
d N=c d \varepsilon+\Phi(|x|) d x
$$

où $c$ et $\Phi$ sont respectivement une constante non-négative et une fonction finie et continue dans $(0,+\infty)$, car $N$ est évidemment absolument continu par rapport à la mesure de Lebegue et sa dénsité est sous-harmonique au sens des distributions en dehors de $O$ et invariante par rotations. Pour une fonction quelconque $f$ de $C_{K}^{+}\left(\boldsymbol{R}^{n}\right)$ invariante par rotations, $N * f$ est bornée sur $\boldsymbol{R}^{n}$ et sous-harmonique dans $\left\{x \in \boldsymbol{R}^{n} ;|x|>\sup _{y \in \operatorname{supp}(f)}|y|\right\}$, Donc $\Phi$ est décroissante au sens large dans $(0,+\infty)$. Par conséquent, pour notre lemme, on peut supposer que $\Phi$ tend vers 0 avec $t \rightarrow+\infty$. $N$ satisfaisant au principe classique du maximum et étant égal à une fonction en dehors de l'origine, $N$ est de type positif (cf. [8]), et par suite $-\Delta N$ est aussi de type positif au sens des distributions. Soit $\sigma$ la restriction de $\Delta N$ en dehors de l'origine; alors $\sigma$ est une mesure de Radon positive en dehors de l'origine invariante par rotations. Pour un nombre positif $r$, on pose $\sigma_{r}$ la restriction de $\sigma$ sur $\left\{x \in \boldsymbol{R}^{n} ;|x| \geqq r\right\}$, et alors elle est considérée comme une mesure de Radon positive dans $\boldsymbol{R}^{n}$. Quelle que soit $\varphi$ de $\mathscr{D}\left(\boldsymbol{R}^{n}\right)$, la fonction $\left(-\Delta N+\sigma_{r}\right) * \varphi * \ddot{\varphi}-\sigma_{r} * \varphi * \ddot{\varphi}$ est de type positif, et par suite

$$
\int \sigma_{r} * \varphi * \stackrel{*}{\varphi} d x \leqq \int\left(-\Delta N+\sigma_{r}\right) * \varphi * \ddot{\varphi} d x<+\infty,
$$


car $\left(-\Delta N+\sigma_{r}\right) * \varphi * \ddot{\varphi}$ est à support compact. Par conséquent, $\int d \sigma_{r}<+\infty$.

D'autre part, soit $\varphi_{1}$ une fonction non-négative de $\mathscr{D}\left(\boldsymbol{R}^{n}\right)$ invariante par rotations telle que $\varphi_{1} \leqq 1$ et $\varphi_{1}(x)=1$ sur $\left\{x \in R^{n} ;|\dot{x}| \leqq 1\right\}$; on pose, pour un entier positif $m, \varphi_{m}(x)=\varphi_{1}(m x)$. On a alors

$$
\lim _{m \rightarrow+\infty} \int \Delta\left(|x|^{2}\left(\varphi_{m}(x)\right)^{2}\right) \Phi(|x|) d x=0,
$$

et par suite, en utilisant la formule de Green,

$$
\begin{aligned}
+\infty> & \int \Delta\left(|x|^{2}\left(\varphi_{1}(x)\right)^{2}\right) \Phi(|x|) d x \\
& =\lim _{m \rightarrow+\infty} \int \Delta\left(|x|^{2}\left(\left(\varphi_{1}(x)\right)^{2}-\left(\varphi_{m}(x)\right)^{2}\right)\right) \Phi(|x|) d x \\
& =\lim _{m \rightarrow+\infty} \int|x|^{2}\left(\left(\varphi_{1}(x)\right)^{2}-\left(\varphi_{m}(x)\right)^{2}\right) d \sigma(x) \geqq \int_{1 \geqq|x|>0}|x|^{2} d \sigma(x),
\end{aligned}
$$

d'où

$$
\int \frac{|x|^{2}}{1+|x|^{2}} d \sigma(x)<+\infty
$$

Par conséquent, on peut écrire

$$
-\widehat{\Delta N}(x)=c^{\prime}+c|x|^{2}+\int(1-\exp (x \cdot y \sqrt{-1})) d \sigma(y),
$$

où $c^{\prime}$ est une autre constante réelle, car $-\Delta N$ est une distribution dans $\boldsymbol{R}^{n}$ invariante par rotations et d'ordre $\leqq 2$. En utilisant encore le fait que $-\Delta N$ est de type positif, on a $c^{\prime} \geqq 0$, d'où $-\widehat{\Delta N}$ est définie-négative dans $\boldsymbol{R}^{n}$.

Si $n \geqq 3,1 / \psi$ est localement sommable dès que $N$ n'est pas constant. Mais, dans le cas où $n=1,2$, cela n'est pas toujours vrai.

On notera $S_{r, 0}=S_{r, 0}\left(\boldsymbol{R}^{n}\right)$ la totalité des noyaux de convolution sur $\boldsymbol{R}^{n}$ complètement sous-harmoniques en dehors de l'origine, invariants par rotations et s'annulant à l'infini.

Remarque 5. Soit $N$ un noyau de convolution borné sur $\boldsymbol{R}^{n}$ complètement sous-harmonique en dehors de l'origine et invariant par rotations; alors il est de la forme $N=N_{0}+c$, où $N_{0}$ est de $S_{r, 0}$ et $c$ est une constante $\geqq 0$.

Le lemme suivant est obtenu par C. S. Herz [4]. 
LEMmE 7. Soit $k$ un entier positif; alors les deux énoncés suivants sont équivalents.

(a). Une distribution $v$ dans $\boldsymbol{R}^{n}$ est k-conditionnellement de type positif.

(b) $v=p_{2 k}(x)+v^{\prime}$, où $p_{2 k}$ est un polynôme homogène de degré $2 k$ et fortement positif et $v^{\prime}$ est conditionnellement de type positif et vérifiant $v^{\prime}=o\left(|x|^{2 k}\right)$ à l'infini.

$v^{\prime}$ est dite conditionnellement de type positif s'il existe un entier $k^{\prime} \geqq 0$ tel que $v^{\prime}$ soit $k^{\prime}$-conditionnellement de type positif. $v^{\prime}=o\left(|x|^{2 k}\right)$ à l'infini signifie que, quelle que soit $\varphi$ de $\mathscr{D}\left(\boldsymbol{R}^{n}\right), v^{\prime} * \varphi=o\left(|x|^{2 k}\right)$ à l'infini.

D'après le lemme 7 , on obtiendra facilement le lemme suivant:

LEMme 8. Soit $N$ un noyau de convolution de $S_{r, 0}\left(\boldsymbol{R}^{n}\right)$. Alors, quel que soit $k$ un entier positif, $\Delta^{k} N$ est $k$-conditionnellement positive.

En effet, posons $\psi=-\widehat{\Delta N}$; alors $\psi$ est définie-négative dans $\boldsymbol{R}^{n}$. On a donc

$$
\Delta^{k} N(x)=(-1)^{k}|x|^{2(k-1)} \psi(x)
$$

On peut écrire

$$
\psi(x)=c_{1}+c_{2}|x|^{2}+\int(1-\exp (x \cdot y \sqrt{-1})) d \sigma(y)
$$

où $c_{i}(i=1,2)$ est une constante non-négative et $\sigma$ est une mesure de Radon positive en dehors de l'origine invariante par rotations avec

$$
\int \frac{|x|^{2}}{1+|x|^{2}} d \sigma(x)<+\infty
$$

Soit $v$ la distribution dans $\boldsymbol{R}^{n}$ définie par la fonction $x \rightarrow(-1)^{k}|x|^{2(k-1)}(\psi(x)$ $\left.-c_{2}|x|^{2}\right)$; alors $v=o\left(|x|^{2 k}\right)$ à l'infini. La distribution $\Delta^{k}\left(N-c_{2} \varepsilon\right)$ vérifie la condition: Quelle que soit $\varphi \geqq 0$ de $\mathscr{D}\left(\boldsymbol{R}^{n}\right)$ avec $\operatorname{supp}(\varphi) \not \partial O$, on a $\Delta^{k}\left(N-c_{2} \varepsilon\right)(\varphi) \geqq 0$. En utilisant le fait que la distribution $\Delta^{k}\left(N-c_{2} \varepsilon\right)$ est d'ordre fini dans un voisinage de l'origine, il existe un entier positif $k^{\prime}$ tel que $\Delta^{k}\left(N-c_{2} \varepsilon\right)$ est $k^{\prime}$-conditionnellement positive, et par suite $v$ est $k^{\prime}$-conditionnellement de type positif, d'après la proposition $7 . \quad v$ est donc $k$-conditionnellement de type positif (cf. le lemme 7). Par conséquent, $\Delta^{k} N=c_{2} \Delta^{k}+\Delta^{k}\left(N-c_{2} \varepsilon\right)$ est $k$-conditionnellement positive.

LEMme 9. Soit $\varphi$ une fonction finie et continue sur $\boldsymbol{R}^{+}$telle que, 
pour un entier positif $n, \varphi(|x|)$ soit définie-négative dans $\boldsymbol{R}^{n}$. Si, pour un entier positif $k$, la fonction $\psi_{n}(x)=(-1)^{k}|x|^{2(k-1)} \varphi(|x|)$ dans $\boldsymbol{R}^{n}$ est $k$ conditionnellement de type positif, alors la fonction $\psi_{1}(t)=(-1)^{k}|t|^{2(k-1)} \varphi(|t|)$ dans $\boldsymbol{R}^{1}$ est aussi k-conditionnellement de type positif.

En effet, soit $f_{1}$ une fonction de $\mathscr{D}^{+}\left(\boldsymbol{R}^{n}\right)$ invariante par rotations telle que

$$
\operatorname{supp}\left(f_{1}\right) \subset\left\{x \in \boldsymbol{R}^{n} ;|x| \leqq 1\right\} \text { et } \int f_{1} d x=1
$$

On pose ensuite, quel que soit $m$ un entier positif, $f_{m}(x)=m^{n} f_{1}(m x)$. En posant

$$
\psi_{1, m}(t)=\psi_{n} * f_{m} * f_{m}(t, 0, \cdots, 0),
$$

on obtient que la suite $\left(\psi_{1, m}\right)_{m=1}^{\infty}$ converge uniformément vers $\psi_{1}$ sur tout compact de $\boldsymbol{R}^{1}$ avec $m \rightarrow+\infty$. $\psi_{n}$ étant $k$-conditionnellement de type positif dans $\boldsymbol{R}^{1}$, la fonction

$$
\psi_{n} *\left(\frac{\partial^{k}}{\partial x_{1}{ }^{k}} f_{m}\right) *\left(\frac{\partial^{k}}{\partial x_{1}{ }^{k}} f_{m}\right)=(-1)^{k} \frac{\partial^{2 k}}{\partial x_{1}{ }^{2 k}}\left(\psi_{n} * f_{m} * f_{m}\right)
$$

est de type positif. La fonction $\psi_{n} * f_{m} * f_{m}$ étant invariante par rotations, on a

$$
\frac{\partial^{2 k}}{\partial x_{1}^{2 k}}\left(\psi_{n} * f_{m} * f_{m}\right)(t, 0, \cdots, 0)=\frac{d^{2 k}}{d t^{2 k}} \psi_{1, m}(t)
$$

pour tout $t$ de $\boldsymbol{R}^{1}$. Par conséquent,

$$
(-1)^{k} \frac{d^{2 k}}{d t^{2 k}} \psi_{1, m}
$$

est de type positif. Faisant $m \rightarrow+\infty$, on obtient que $(-1)^{k}\left(d^{2 k} / d t^{2 k}\right) \psi_{1}$ est de type positif au sens des distributions dans $R^{1}$, d'où, quelle que soit $f$ de $\mathscr{D}\left(\boldsymbol{R}^{1}\right)$,

$$
\psi_{1} *\left(\frac{d^{k}}{d t^{k}} f\right) *\left(\frac{d^{*}}{d t^{k}} f\right)(0) \geqq 0 .
$$

On remarque ici que, dans $\boldsymbol{R}^{1}$,

$$
\theta_{0}^{k}=\left\{\frac{d^{k}}{d t^{k}} f ; f \in \mathscr{D}\left(\boldsymbol{R}^{1}\right)\right\},
$$


qui est facilement montré. C. S. Herz a montré sa forme générale (cf. [4]). Par conséquent, $\psi_{1}$ est $k$-conditionnellement de type positif.

Dans cette note, on notera toujours $G_{0}^{(n)}$ ou bien $r^{2-n}$ le noyau newtonien. Dans ce cas, on peut supposer $\widehat{G_{0}^{(n)}}=1 /|x|^{2}$. Pour tout $p>0$, il existe le noyau de convolution de Dirichlet $G_{p}^{(n)}$ sur $\boldsymbol{R}^{n}(n \geqq 1)$ dont la transformée de Fourier est de la forme

$$
\widehat{G_{p}^{(n)}}(x)=\frac{1}{p+|x|^{2}}
$$

La famille $\left(G_{p}^{(n)}\right)_{p>0}$ est évidemment une résolvante. On a $\lim _{p \rightarrow 0} G_{p}^{(n)}=G_{0}^{(n)}$ sur $\boldsymbol{R}^{n}(n \geqq 3)$, et dans ce cas, $\left(G_{p}^{(n)}\right)_{p \geqq 0}$ s'appelle la résolvante associée au noyau newtonien.

THÉORÈme 2. (1) Soit $N$ un noyau de convolution borné sur $\boldsymbol{R}^{n}$ complètement sous-harmonique en dehors de l'origine et invariant par rotations; alors il est de la forme

$$
N=c_{1}+c_{2} \varepsilon+\int G_{p}^{(n)} d \lambda(p),
$$

où $c_{i}(i=1,2)$ et $\lambda$ sont respectivement une constante non-négative et une mesure de Radon positive sur $\boldsymbol{R}^{+}$, et $N$ satisfait au principe complet du maximum.

(2) Dans $\boldsymbol{R}^{n}(n \geqq 3), S_{r, 0}\left(\boldsymbol{R}^{n}\right)$ est un seul cône convexe de Riesz relatif au noyau newtonien sur $R^{n}$ et constitué par de noyaux de convolution invariants par rotations.

Démonstration. On montrera d'abord l'énoncé (1). D'après le lemme 6 , il existe une fonction non-négative, finie et continue $\varphi$ sur $\boldsymbol{R}^{+}$telle que la fonction

$$
-\widehat{\Delta N}(x)=\varphi(|x|)
$$

soit définie-négative dans $\boldsymbol{R}^{n}$. Posons $\psi_{1}(t)=\varphi(|t|)$ sur $\boldsymbol{R}^{1}$; alors $\psi_{1}$ est définie-négative dans $\boldsymbol{R}^{1}$. Pour tout l'entier positif $k, \Delta^{k} N$ est $k$-conditionnellement positive, et donc la fonction

$$
\psi_{n, k}(x)=(-1)^{k}|x|^{2(k-1)} \varphi(|x|)
$$

dans $\boldsymbol{R}^{n}$ est $k$-conditionnellement de type positif (au sens des distributions). D'après le présent lemme, la fonction 


$$
\psi_{1, k}(t)=(-1)^{k}|t|^{2(k-1)} \varphi(|t|)
$$

est $k$-conditionnellement de type positif dans $\boldsymbol{R}^{1}$. Soit $u$ la distribution 1-conditionnellement positive dans $\boldsymbol{R}^{1}$ dont la transformée de Fourier est égale à $-\psi_{1}$. Ayant, quel que soit $k$ un entier non-négatif,

$$
\frac{\widehat{d^{2 k}}}{d t^{2 k}} u(t)=(-1)^{k+1}|t|^{2 k} \psi_{1}(t),
$$

on obtient que $\left(d^{2 k} / d t^{2 k}\right) u$ est $(k+1)$-conditionnellement positive. Donc il existe une fonction non-négative et infiniment dérivable $\varphi_{1}$ dans $(0,+\infty)$ telle que $u=\varphi_{1}(|t|) d t$ en dehors de l'origine et, quel que soit $k$ un entier non-négatif, $\left(d^{2 k} / d t^{2 k}\right) \varphi_{1}(t) \geqq 0$ dans $(0,+\infty)$. $\psi_{1}$ étant définie-négative, on peut écrire

$$
\psi_{1}(t)=c_{1}+c_{2} t^{2}+\int(1-\exp (t s \sqrt{-1})) d \sigma(s),
$$

où $c_{i}(i=1,2)$ et $\sigma$ sont respectivement une constante non-négative et une mesure de Radon positive en dehors de l'origine avec $\int \frac{t^{2}}{1+t^{2}} d \sigma(t)$ $<+\infty$. On a $u=\sigma$ en dehors de l'origine, et par suite

$$
\int \frac{t^{2}}{1+t^{2}} \varphi_{1}(|t|) d t<+\infty
$$

On a donc $\lim _{t \rightarrow+\infty} \varphi_{1}(t)=0$. D'après le théorème de Bernstein et le lemme 5 , il existe une mesure de Radon positive $\lambda$ sur $\boldsymbol{R}^{+}$telle que

$$
\varphi_{1}(t)=\int \exp (-t s) d \nu(s) \quad(\forall t>0) .
$$

D'après $\lim _{t \rightarrow+\infty} \varphi_{1}(t)=0$, on a $\nu(\{0\})=0$. On a donc

$$
\begin{aligned}
\int(1- & \exp (t s \sqrt{-1})) \varphi_{1}(|s|) d s \\
= & \int\left(\int_{s>0}(1-\exp (t s \sqrt{-1})) \exp (-s r) d s\right. \\
& \left.\quad+\int_{s<0}(1-\exp (t s \sqrt{-1})) \exp (s r) d s\right) d \nu(r) \\
= & 2 \int\left(\frac{1}{s}-\frac{\frac{1}{2}}{s-t \sqrt{-1}}-\frac{\frac{1}{2}}{s+t \sqrt{-1}}\right) d \nu(s)=2 \int\left(1-\frac{s^{2}}{s^{2}+t^{2}}\right) \frac{1}{s} d \nu(s) \\
= & 2 \int\left(1-s^{2} \widehat{G_{s^{2}}^{(1)}}(t)\right) \frac{1}{s} d \nu(s) .
\end{aligned}
$$


Par conséquent, on a

$$
\psi_{1}(t)=c_{1}+c_{2} t^{2}+2 \int\left(1-s^{2} \widehat{G_{s^{2}}^{(1)}}(t)\right) \frac{1}{s} d \nu(s),
$$

et par suite

$$
-\widehat{\Delta N}(x)=c_{1}+c_{2}|x|^{2}+2 \int\left(1-s^{2} \widehat{G_{s^{2}}^{(n)}}(x)\right) \frac{1}{s} d \nu(s)
$$

dans $\boldsymbol{R}^{n}$. Soit $N_{0}$ le noyau de convolution sur $\boldsymbol{R}^{n}$ de $S_{r, 0}$ tel que $N-N_{0}$ soit constant (cf. la remarque 5). Alors la transformée de Fourier de $N_{0}$ est égale à une fonction et on a

$$
\begin{aligned}
\hat{N}_{0}(x) & =\frac{c_{1}}{|x|^{2}}+c_{2}+\frac{2}{|x|^{2}} \int\left(1-s^{2} \widehat{G_{s^{2}}^{(n)}}(x)\right) \frac{1}{s} d \nu(s) \\
& =\frac{c_{1}}{|x|^{2}}+c^{2}+2 \int \widehat{G_{s^{2}}^{(n)}}(x) \frac{1}{s} d \nu(s) .
\end{aligned}
$$

Si $n=1,2$, alors $c_{1}=0$, car $\hat{N}_{0}$ est localement summable. Soit $\lambda$ la mesure de Radon positive sur $\boldsymbol{R}^{+}$définie par

$$
\int f d \lambda=2 \int f\left(t^{2}\right) \frac{1}{t} d \nu(t)+c_{1} f(0)
$$

pour toute la fonction $f$ de $C_{K}\left(\boldsymbol{R}^{+}\right)$. Posons encore $c_{1}=N-N_{0}$; alors

$$
N=c_{1}+c_{2} \varepsilon+\int G_{s}^{(n)} d \lambda(s)
$$

D'après la proposition $1, N_{0}$ est un noyau de convolution de Dirichlet sur $\boldsymbol{R}^{n}$ dès que $N_{0} \neq 0$, et par suite $N$ satisfait au principe complet du maximum. La démonstration de (1) est ainsi complète.

Avant la démonstration de l'énoncé (2), on préparera le lemme suivant:

LEMME 10. Soit $C\left(r^{2-n}\right)$ un cône convexe de Riesz relatif au noyau newtonien sur $\boldsymbol{R}^{n}(n \geqq 3)$. Alors tout l'élément de $C\left(r^{2-n}\right)$ est complètement sous-harmonique en dehors de l'origine et s'annulant à l'infini.

En effet, d'après la remarque 1 , on a, quel que soit $N$ de $C\left(r^{2-n}\right)$, $N \prec G_{0}^{(n)}$, et par suite, $N$ s'annule à l'infini. Soit $N$ un noyau de convolution de Hunt quelconque sur $X$ contenu dans $C\left(r^{2-n}\right)$; on désigne par $N^{\prime}$ son noyau dual relatif au noyau newtonien. Alors on a $(-\Delta N) * N^{\prime}=\varepsilon$. 
$N^{\prime}$ étant un noyau de convolution de Hunt sur $\boldsymbol{R}^{n}$, on a $N^{\prime}=\int_{0}^{\infty} \alpha_{t}^{\prime} d t$, où $\left(\alpha_{t}^{\prime}\right)_{t \geqq 0}$ est un semi-groupe vaguement continu de mesures de Radon positives dans $X$ avec $\int d \alpha_{t}^{\prime} \leqq 1$. Donc

$$
\lim _{t \rightarrow 0} \frac{\varepsilon-\alpha_{t}^{\prime}}{t}=-\Delta N
$$

au sens des distributions dans $\boldsymbol{R}^{n}$, d'où $\Delta N \geqq 0$ au sens des distributions en dehors de l'origine. Soit $m$ un entier positif et supposons que, quel que soit $N$ de $C\left(r^{2-n}\right), \Delta^{m} N \geqq 0$ au sens des distributions en dehors de l'origine. Soit $N$ un noyau de convolution de Hunt de $C\left(r^{2-n}\right)$; on désigne ensuite par $N^{\prime}$ le noyau dual de $N$ relatif au noyau newtonien, et par $\left(N_{p}^{\prime}\right)_{p \geqq 0}$ la résolvante associée au noyau $N^{\prime}$. On a alors $\left(N_{p}^{\prime}\right)_{p \geqq 0} \subset C\left(r^{2-n}\right)$, d'après la proposition 5. Ayant, quel que soit $p>0$,

$$
\left(p N_{p}^{\prime}\right) * N=p N * N^{\prime}-p^{2} N * N^{\prime} * N_{p}^{\prime}=p G_{0}^{(n)}-p^{2} G_{0}^{(n)} * N_{p}^{\prime},
$$

on obtient donc

$$
\Delta^{m+1}\left(p N_{p}^{\prime}\right) * N=-p \Delta^{m}+p^{2} \Delta^{m} N_{p}^{\prime}
$$

au sens des distributions dans $\boldsymbol{R}^{n}$. D'après notre hypothèse, on a

$$
\Delta^{m+1}\left(p N_{p}^{\prime}\right) * N \geqq 0
$$

au sens des distributions en dehors de l'origine. D'après $p \int d N_{p}^{\prime} \leqq 1$ $\left(\forall p>0\right.$ ) et $\lim _{p \rightarrow+\infty} p N_{p}^{\prime}=\varepsilon$ (au sens de la topologie vague), on a $\Delta^{m+1} N \geqq 0$ au sens des distributions en dehors de l'origine. Par récurrence $N$ est complètement sous-harmonique en dehors de l'origine, d'où notre lemme.

Montrons (2) dans le théorème 2. D'après le théorème 1 , la totalité $C_{R}\left(r^{2-n}\right)$ des noyaux de convolution $N$ sur $\boldsymbol{R}^{n}$ de la forme $N=c \varepsilon+\int G_{p}^{(n)} d \lambda(p)$ est un cône convexe de Riesz relatif au noyau newtonien, où $c$ et $\lambda$ sont respectivement une constante $\geqq 0$ et une mesure de Radon positive sur $R^{+}$. Soit $C\left(r^{2-n}\right)$ un cône convexe de Riesz quelconque relatif au noyau newtonien sur $\boldsymbol{R}^{n}$ et constitué par noyaux de convolution invariants par rotations. D'après l'énoncé (1), on a $S_{r, 0} \subset C_{R}\left(r^{2-n}\right)$, et d'après la remarque 3, $C_{R}\left(r^{2-n}\right) \subset C\left(r^{2-n}\right)$. En utilisant finalement le lemme 10, $C\left(r^{2-n}\right) \subset S_{r, 0}$, d'où $S_{r, 0}=C\left(r^{2-n}\right)$. La démonstration est ainsi complète. 
Remarque 6. Pour un noyau de convolution de Hunt borné $\kappa$ sur $\boldsymbol{R}$ porté par $\boldsymbol{R}^{+}$et pour un nombre $p>0$, le noyau de convolution

$$
N_{(s, p)}=\int \alpha_{t} \exp (-p t) d \kappa(t)
$$

sur $\boldsymbol{R}^{n}$ est aussi un noyau de convolution de Dirichlet sur $\boldsymbol{R}^{n}$, où $\left(\alpha_{t}\right)_{t \geqq 0}$ est le semi-groupe associé au noyau newtonien, car le noyau de convolution $\kappa_{(p)}$ sur $\boldsymbol{R}$ satisfait au principe complet du maximum, où $d \kappa_{(p)}(t)$ $=\exp (-p t) d_{\kappa}(t)$. Est-ce qu'une discussion analogue pour $\left(N_{(s, p)}\right)_{p \geqq 0}$ a lieu?

Nous discuterons finalement sur une généralisation du théorème de Bernstein.

Soit $C^{\infty}(0,+\infty)$ l'espace vectoriel topologique des fonctions réelles et infiniment dérivables dans $(0,+\infty)$ muni des semi-normes

$$
p_{m, k}(f)=\sup _{1 / m \leqq t \leqq m}\left|f^{(k)}(t)\right| \quad(m=1,2, \ldots ; k=0,1,2, \cdots) .
$$

Rappelons le théorème de Krein-Milman et le lemme 5. Alors le théorème de Bernstein affirme l'éconcé suivant:

Le sous-ensemble

$$
\begin{aligned}
C M_{1}= & \left\{f \in C^{\infty}(0,+\infty) ; f^{(2 k)}(t) \geqq 0\right. \\
& \text { dans } \left.(0,+\infty), \varlimsup_{t \rightarrow 0} f(t) \leqq 1, \varlimsup_{t \rightarrow+\infty} f(t)<+\infty\right\}
\end{aligned}
$$

est convexe et compact dans $C^{\infty}(0,+\infty)$, et on a

$$
\text { ex. } C M_{1}=\left\{g_{p} ; 0 \leqq p \leqq \infty\right\},
$$

où ex. $C M_{1}$ est l'ensemble des points extrêmes de $C M_{1}, g_{p}(t)=\exp (-\sqrt{p} t)$ $(0 \leqq p<\infty)$ et $g_{\infty}(t)=0$.

DÉFINITION 6. Soit $\alpha$ un entier non-négatif. Une fonction $f$ de $C^{\infty}(0,+\infty)$ sera dite compètement monotone d'ordre $\alpha$ si l'on a, quel que soit $k$ un entier non-négatif,

$$
\left(B_{\alpha}\right)^{k}\left(t ; \frac{d}{d t}\right) f(t) \geqq 0 \quad \text { dans } \quad(0,+\infty) \text { et } \varlimsup_{t \rightarrow+\infty} f(t)<+\infty,
$$

où

$$
B_{\alpha}\left(t ; \frac{d}{d t}\right)=\frac{d^{2}}{d t^{2}}-\frac{\alpha}{t} \frac{d}{d t}, \quad B_{\alpha}^{0}\left(t ; \frac{d}{d t}\right) f=f
$$


et

$$
B_{\alpha}^{k+1}\left(t ; \frac{d}{d t}\right)=B_{\alpha}\left(t ; \frac{d}{d t}\right)\left(B_{\alpha}^{k}\left(t ; \frac{d}{d t}\right)\right)
$$

On notera $C M_{\alpha, 1}$ la totalité des fonctions complètement monotones d'ordre $\alpha$ dans $(0,+\infty)$ telles que $\varlimsup_{\lim }+\infty f(t) \leqq 1$. En utilisant la fonction besselien, on obtient que, pour tout le nombre $p \geqq 0$, il existe une fonction $g_{p}^{(\alpha)}$ de $C M_{\alpha, 1}$, et une seule telle que

$$
B_{\alpha}\left(t ; \frac{d}{d t}\right) g_{p}^{(\alpha)}=p g_{p}^{(\alpha)} \text { et } \lim _{t \rightarrow 0} g_{p}^{(\alpha)}(t)=1 \text {. }
$$

On a évidemment $g_{0}^{(\alpha)}=1$. Il est évident que $g_{p}^{(0)}=g_{p}\left({ }^{\forall} p \geqq 0\right)$. Cela est, d'autre part, compris par

$$
\Delta\left(\frac{\varphi(|x|)}{|x|^{\alpha+1}}\right)=\frac{B_{\alpha}(|x| ; d / d t) \varphi(|x|)}{|x|^{\alpha+1}}
$$

dans $R^{\alpha+3}-\{0\}$, où $\varphi$ et $\Delta$ sont respectivement une fonction de $C^{\infty}(0,+\infty)$ et le laplacien sur $\boldsymbol{R}^{\alpha+3}$.

LEMME 11. Soient $\alpha$ un entier non-négatif et $f$ une fonction nonnégative de classe $C^{2}$ dans $(0,+\infty)$ telle que $B_{\alpha}(t ; d / d t) f(t) \geqq 0$ dans $(0,+\infty)$ et $\varlimsup_{\lim }++\infty f(t)<+\infty$; alors $f$ est décroissante (au sens large).

En effet, posons $n=\alpha+3$. On a

$$
\Delta\left(\frac{f(|x|)}{|x|^{n-2}}\right)=\frac{B_{\alpha}(|x| ; d / d t) f(|x|)}{|x|^{n-2}}
$$

dans $\boldsymbol{R}^{n}-\{0\}$. Donc la fonction $f(|x|) /|x|^{n-2}$ est sous-harmonique en dehors de l'origine. Elle tendant vers 0 avec $|x| \rightarrow+\infty$, il est facile de voir que $f$ est décroissante (au sens large).

Par conséquent, pour toute la fonction $f$ complètement monotone d'ordre $\alpha$, la limite $\lim _{t \rightarrow 0} f(t)$ existe, qui notera $f(0)=\lim _{t \rightarrow 0} f(t)$. En généralisant le théorème de Bernstein, on obtient le théorème suivant:

THÉORÈME 3. Soit $\alpha$ un entier non-négatif. Alors $C M_{\alpha, 1}$ est un sousensemble convexe et compact de $C^{\infty}(0,+\infty)$, et l'ensemble des points extrêmes ex. $C M_{\alpha, 1}$ de $C M_{\alpha, 1}$ est égal à $\left\{g_{p}^{(\alpha)} ; 0 \leqq p \leqq+\infty\right\}$, où $g_{\infty}^{(\alpha)}=0$.

Démonstration. Evidemment $C M_{\alpha, 1}$ est convexe. Pour que $C M_{\alpha, 1}$ soit compact, il suffit qu'il soit fermé et borné dans $C^{\infty}(0,+\infty)$. On rappelle 
ici le théorème d'Ascoli. Il est facile de voir qu'il est fermé. Montrons que $C M_{\alpha, 1}$ est borné dans $C^{\infty}(0,+\infty)$. On a, quels que soient $m$ un entier $\geqq 1$ et $f$ une fonction de $C M_{\alpha, 1}, p_{m, 0}(f) \leqq 1$. Pour simplifier la notation, on pose $n=\alpha+3$. Pour tout l'entier non-négatif $k$ et pour toute la fonction $f$ de $C M_{\alpha, 1}$, la fonction

$$
\frac{B_{\alpha}^{k}(|x| ; d / d t) f(|x|)}{|x|^{n-2}}
$$

est sous-harmonique dans $\boldsymbol{R}^{n}-\{0\}$. On a, quelle que soit $\varphi \geqq 0$ de $\mathscr{D}\left(\boldsymbol{R}^{n}\right)$,

$$
\lim _{|x| \rightarrow+\infty} \int \frac{B_{\alpha}^{k}(|x-y| ; d / d t) f(|x-y|)}{|x-y|^{n-2}} \varphi(y) d y=\lim _{|x| \rightarrow+\infty} \int \frac{f(|x-y|)}{|x-y|^{n-2}} \Delta^{k} \varphi(y) d y=0
$$

et donc

$$
\lim _{|x| \rightarrow+\infty} \frac{B_{\alpha}^{k}(|x| ; d / d t) f(|x|)}{|x|^{n-2}}=0
$$

Par conséquent, $B_{\alpha}^{k}(t ; d / d t) f(t)$ est décroissante (au sens large) dans $(0,+\infty)$.

Soit $k$ un entier quelconque $\geqq 1$, et supposons que, pour un entier quelconque $j$ avec $0 \leqq j \leqq 2 k$ et pour un entier quelconque $m \geqq 1$, il existe une constante $c_{m, j}>0$ telle que, quelle que soit $f$ de $C M_{\alpha, 1}, p_{m, j}(f) \leqq c_{m, j}$. Alors, pour un entier quelconque $j$ avec $0 \leqq j \leqq k$, il existe un autre constante positive $a_{m, j}$ telle que, quelle que soit $f$ de $C M_{\alpha, 1}$,

$$
\sup _{1 / m \leqq t \leqq m}\left|B_{a}^{j}\left(t ; \frac{d}{d t}\right) f(t)\right| \leqq a_{m, j} .
$$

Soit $m$ un entier positif fixé. Alors il existe une constante $c_{m}$ avec $0<c_{m}<1$ telle que

$$
\begin{aligned}
\varphi_{m}(x)= & \int G_{0}^{(n)}(x-y) d s_{1 / 2 m}(y)-c_{m} \int G_{0}^{(n)}(x-y) d s_{1 / 4 m}(y)-\left(1-c_{m}\right) \\
& \int G_{0}^{(n)}(x-y) d s_{1 / m}(y)
\end{aligned}
$$

soit zéro sur $\left\{x \in \boldsymbol{R}^{n} ;|x| \leqq 1 / 4 m\right.$ ou $\left.|x| \geq 1 / m\right\}$, où, pour $r>0$, $s_{r}$ désigne la mesure uniforme sur $\left\{x \in \boldsymbol{R}^{n} ;|x|=r\right\}$ de masse totale d'unité. On a, dans ce cas, $\varphi_{m}(x)>0$ dans $\left\{x \in R^{n} ; 1 / 4 m<|x|<1 / m\right\}$. On a ensuite, quelle que soit $f$ de $C M_{\alpha, 1}$, 


$$
\begin{aligned}
& \int \frac{B_{\alpha}^{k+1}(|x| ; d / d t) f(|x|)}{|x|^{n-2}} \varphi_{m}(x) d x \\
& =c_{m} \int \frac{B_{\alpha}^{k}(|x| ; d / d t) f(|x|)}{|x|^{n-2}} d s_{1 / 4 m}(x)+\left(1-c_{m}\right) \int \frac{B_{\alpha}^{k}(|x| ; d / d t) f(|x|)}{|x|^{n-2}} d s_{1 / m}(x) \\
& \quad-\int \frac{B_{\alpha}^{k}(|x| ; d / d t) f(|x|)}{|x|^{n-2}} d s_{1 / 2 m}(x) \\
& =c_{m}(4 m)^{n-2} B_{\alpha}^{k}\left(\frac{1}{4 m} ; \frac{d}{d t}\right) f\left(\frac{1}{4 m}\right)+\left(1-c_{m}\right) m^{n-2} B_{a}^{k}\left(\frac{1}{m} ; \frac{d}{d t}\right) f\left(\frac{1}{m}\right) \\
& \quad-(2 m)^{n-2} B_{\alpha}^{k}\left(\frac{1}{2 m} ; \frac{d}{d t}\right) f\left(\frac{1}{2 m}\right) \\
& \leqq \\
& \quad(4 m)^{n-2} a_{4 m, k},
\end{aligned}
$$

car

$$
\Delta \varphi_{m}=c_{m} s_{1 / 4 m}+\left(1-c_{m}\right) s_{1 / m}-s_{1 / 2 m}
$$

au sens des distributions dans $\boldsymbol{R}^{n}$. On a donc

$$
\left(\int \varphi_{m} d x\right) B_{\alpha}^{k+1}\left(\frac{1}{m} ; \frac{d}{d t}\right) f\left(\frac{1}{m}\right) \leqq(4 m)^{n-2} a_{4 m, k} .
$$

La fonction $B_{\alpha}^{k+1}(t, d / d t) f(t)$ étant décroissante dans $(0,+\infty)$, pour tout l'entier $m \geqq 1$, il existe une constante positive $a_{m, k+1}$ telle que, quelle que soit $f$ de $C M_{\alpha, 1}$,

$$
\sup _{1 / m \leqq t \leqq m}\left|B_{\alpha}^{k+1}\left(t, \frac{d}{d t}\right) f(t)\right| \leqq a_{m, k+1}
$$

On a, pour une fonction $f$ de $C M_{\alpha, 1}$,

$$
\frac{d}{d t}\left(\frac{d}{d t} B_{\alpha}^{k}\left(t ; \frac{d}{d t}\right) f(t)-\frac{\alpha}{t} B_{\alpha}^{k}\left(t ; \frac{d}{d t}\right) f(t)\right) \geqq 0
$$

et

$$
\frac{d}{d t} B_{\alpha}^{k}\left(t ; \frac{d}{d t}\right) f(t)-\frac{\alpha}{t} B_{\alpha}^{k}\left(t ; \frac{d}{d t}\right) f(t) \leqq 0
$$

Done

$$
\begin{aligned}
& \frac{1}{2 m} \sup _{1 / m \leqq t \leqq m}\left|\frac{d}{d t} B_{\alpha}^{k}\left(t ; \frac{d}{d t}\right) f(t)-\frac{\alpha}{t} B_{\alpha}^{k}\left(t ; \frac{d}{d t}\right) f(t)\right| \\
& =\frac{1}{2 m}\left(-\frac{d}{d t} B_{\alpha}^{k}\left(\frac{1}{m} ; \frac{d}{d t}\right) f\left(\frac{1}{m}\right)+m \alpha B_{\alpha}^{k}\left(\frac{1}{m} ; \frac{d}{d t}\right) f\left(\frac{1}{m}\right)\right)
\end{aligned}
$$




$$
\begin{aligned}
& \leqq B_{\alpha}^{k}\left(\frac{1}{2 m} ; \frac{d}{d t}\right) f\left(\frac{1}{2 m}\right)+\left(\frac{\alpha}{2}-1\right) B_{\alpha}^{k}\left(\frac{1}{m} ; \frac{d}{d t}\right) f\left(\frac{1}{m}\right) \\
& \leqq\left(1+\frac{\alpha}{2}\right) a_{2 m, k}
\end{aligned}
$$

et par suite

$$
\sup _{1 / m \leqq t \leqq m}\left|\frac{d}{d t} B_{\alpha}^{k}\left(t ; \frac{d}{d t}\right) f(t)\right| \leqq 2 m^{2} \alpha a_{m, k}+2 m\left(1+\frac{\alpha}{2}\right) a_{2 m, k} .
$$

On a, en même temps,

$$
\sup _{f \in C M_{\alpha, 1}}\left(\sup _{1 / m \leqq t \leqq m}\left|\frac{d^{2}}{d t^{2}} B_{\alpha}^{k}\left(t ; \frac{d}{d t}\right) f(t)\right|\right)<+\infty .
$$

On obtient ainsi que, quel que soit $m$ un entier $>1$,

$$
\sup _{f \in C M_{\alpha, 1}} p_{m, 2 k+1}(f)<+\infty \text { et } \sup _{f \in C M_{\alpha, 1}} p_{m, 2(k+1)}(f)<+\infty \text {. }
$$

Par récurrence, $C M_{\alpha, 1}$ est borné dans $C^{\infty}(0,+\infty)$, d'où $C M_{\alpha, 1}$ est un sousensemble convexe et compact de $C^{\infty}(0,+\infty)$.

Montrons ensuite ex. $C M_{\alpha, 1}=\left\{g_{p}^{(\alpha)} ; 0 \leqq p \leqq+\infty\right\}$. Rappelons encore que, quelle que soit $\varphi$ de $C^{\infty}(0,+\infty)$,

$$
\Delta\left(\frac{\varphi(|x|)}{|x|^{n-2}}\right)=\frac{B_{\alpha}(|x| ; d / d t) \varphi(|x|)}{|x|^{n-2}}
$$

On a alors

$$
G_{p}^{(n)}=\frac{g_{p}^{(\alpha)}(|x|)}{|x|^{n-2}} d x \quad(\forall p \geqq 0) .
$$

Soit $f$ une fonction non-zéro de ex. $C M_{\alpha, 1}$. Alors on a évidemment $f(0)=1$. Le noyau de convolution $\left(f(|x|) /|x|^{n-2}\right) d x$ sur $\boldsymbol{R}^{n}$ appartenant à $S_{r, 0}\left(\boldsymbol{R}^{n}\right)$, il existe une mesure de Radon positive $\lambda$ dans $[0,+\infty)$ de masse totale d'unité telle que

$$
f=\int g_{p}^{(\alpha)} d \lambda(p)
$$

Supposons que $\lambda$ n'est pas une mesure d'unité à un point. Alors il existe un nombre $t>0$ tel que $\lambda([0, t))>0$ et $\lambda([t,+\infty))>0$. Soient $\lambda_{1}$ et $\lambda_{2}$ les restrictions de $\lambda \operatorname{sur}[0, t)$ et sur $[t,+\infty)$, respectivement. On a alors 


$$
f \pm\left(\int d \lambda_{2} \int g_{p}^{(\alpha)} d \lambda_{1}(p)-\int d \lambda_{1} \int g_{p}^{(\alpha)} d \lambda_{2}(p)\right) \in C M_{\alpha, 1}
$$

et par suite

$$
\frac{1}{\int d \lambda_{1}} \int g_{p}^{(\alpha)} d \lambda_{1}(p)=\frac{1}{\int d \lambda_{2}} \int g_{p}^{(\alpha)} d \lambda_{2}(p)
$$

Mais cela est en contradiction avec $g_{p}^{(\alpha)}>g_{q}^{(\alpha)}$ dans $(0,+\infty)$ dès que $p<q$, d'où $\lambda$ est une mesure d'unité à un point. Par conséquent, ex. $C M_{\alpha, 1}-\{0\}$ $\subset\left\{g_{p}^{(\alpha)} ; 0 \leqq p<+\infty\right\}$. On montrera l'inclusion inverse. Supposons que l'inclusion inverse n'a pas lieu; alors il existe un nombre $q>0$ et une mesure de Radon positive $\lambda \neq \varepsilon_{q}$ dans $[0,+\infty)$ de masse totale d'unité tels que

$$
g_{q}^{(\alpha)}=\int g_{p}^{(\alpha)} d \lambda(p)
$$

et donc

$$
G_{q}^{(n)}=\int G_{p}^{(n)} d \lambda(p)
$$

On a alors

$$
\frac{1}{t^{2}+q}=\int \frac{1}{t^{2}+p} d \lambda(p)
$$

sur $R^{1}$, et par suite

$$
\exp (-\sqrt{q} t)=\int \exp (-\sqrt{p} t) d \lambda(p) \quad(\forall t \geqq 0),
$$

d'après l'injectivité de la transformation de Fourier. Mais cette égalité est en contradiction avec l'injectivité de la transformation de Laplace. Par conséquent, l'inclusion inverse a lieu. Il est évident que 0 est extrême. La démonstration est ainsi complète.

COROLlaIRE 1. Soient $\alpha$ un entier non-négatif et $\varphi$ une fonction de $C^{\infty}(0,+\infty)$. Pour que $\varphi$ soit complètement monotone d'ordre $\alpha$, il faut et il suffit qu'il existe une mesure de Radon positive $\lambda$ sur $[0,+\infty)$ telle que 


$$
\varphi=\int g_{p}^{(\alpha)} d \lambda(p)
$$

Dans.ce cas, $\lambda$ est uniquement déterminée.

Cela résulte immédiatement des théorèmes 2 et 3.

CoRollaire 2. Soit $S_{r, 0}^{(1)}$ la totalité des noyaux de convolution $N$ sur $\boldsymbol{R}^{n}(n \geqq 3)$ complètement sous-harmoniques en dehors de l'origine, invariants par rotations, s'annulant à l'infini et vérifiant $N(\{O\})=0$ et $\varlimsup_{\lim } N(x)|x|^{n-2} \leqq 1^{(3)}$. Alors $S_{r, 0}^{(1)}$ est convexe et compact dans $C^{\infty}\left(\boldsymbol{R}^{n}-\{0\}\right)$ et on $a$

$$
\text { ex. } S_{r, 0}^{(1)}=\left\{G_{p}^{(n)} ; 0 \leqq p \leqq+\infty\right\}
$$

où $G_{\infty}^{(n)}=0$, où $C^{\infty}\left(\boldsymbol{R}^{n}-\{0\}\right)$ est l'espace de Fréchet usuel des fonctions réelles et infiniment dérivables en dehors de l'origine et où ex. $S_{r, 0}^{(1)}$ est l'ensemble des points extrêmes de $S_{r, 0}^{(1)}$.

Cela est un résultat immédiat du théorème 3.

\section{BIBLIOGRAPHIES}

[1] A. Beurling et J. Deny: Dirichlet spaces, Proc. Nat. Acad. Sc. U.S.A., 45 (1959), 208-215.

[2] N. Bourbaki: Espaces vectoriels topologiques, Hermann, Paris, 1966.

[ 3 ] J. Deny: Noyaux de convolution de Hunt et noyaux associés à une famille fondamentale, Ann. Inst. Fourier (Grenoble), 12 (1962), 643-667.

[4] C. S. Herz: Analyse harmonique à plusieurs variables, Sém. Math. d'Orsay, $1965 / 66$.

[5] I. Higuchi et M. Itô: Characterization of the relative domination principle, Nagoya Math. J., 50 (1972), 175-184.

[6] M. Itô: Remarque sur la somme d'un noyau de Dirichlet et du noyau newtonien, Proc. Japan Acad., 46 (1970), 362-363.

[ 7 ] _ : Remarque sur la somme des résolvantes, Proc. Japan Acad., 46 (1970), 243-245.

[8] — : Sur les principles divers du maximum et le type positif, Nagoya Math. J., 44 (1971), 133-164.

[9] — : Caractérisation du principe de domination pour les noyaux de convolution, Nagoya Math. J., à paraître.

[10] — : Sur la famille sous-ordonnée au noyau de convolution de Hunt, Nagoya Math. J., 51 (1973), 45-56.

[11] —-: Sur la famille sous-ordonnée au noyau de convolution de Hunt II, Nagoya Math. J., 53 (1974), 115-126.

(3) $N$ est considéré comme une fonction infiniment dérivable en dehors l'origine. 
[12] M. Riesz: Intégrales de Riemann-Lioville et potentiels, Acta Sc. Math., Szeged, 9 (1938), 1-42.

[13] D. Widder: The Laplace transform, Princeton Univ. Press, Princeton, 1948.

Institut Mathématique

d'Université de Nagoya 